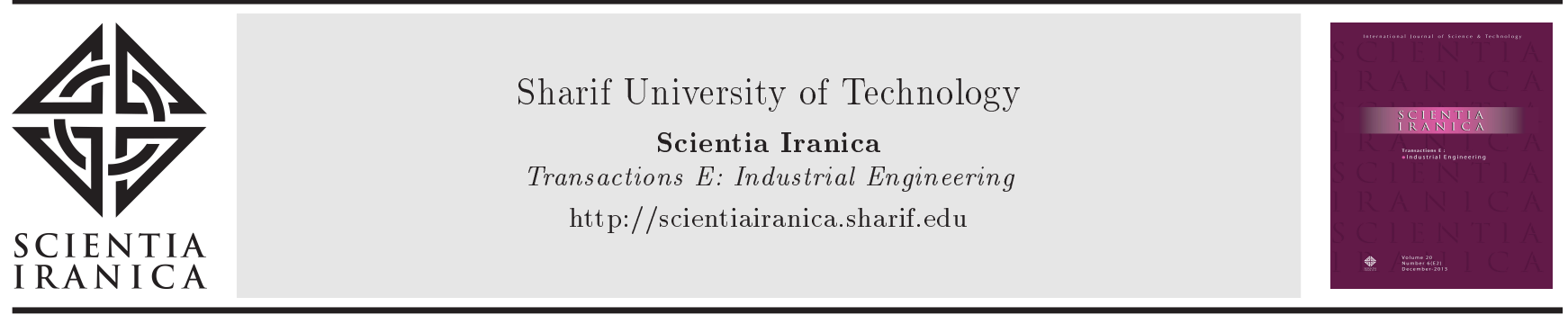

\title{
Extended TOPSIS method for multi-criteria group decision-making problems under cubic intuitionistic fuzzy environment
}

\author{
H. Garg* and G. Kaur \\ School of Mathematics, Thapar Institute of Engineering \& Technology (Deemed University), Patiala 147004, Punjab, India.
}

Received 2 October 2017; received in revised form 20 June 2018; accepted 4 August 2018

\author{
KEYWORDS \\ Cubic intuitionistic \\ fuzzy sets; \\ IVIFS; \\ TOPSIS method; \\ Distance measures; \\ Closeness coefficients; \\ Multi-criteria group \\ decision-making.
}

\begin{abstract}
The objective of this work is to present a novel Multi-Criteria Group DecisionMaking (MCGDM) method under the Cubic Intuitionistic Fuzzy (CIF) environment by integrating it with the extended TOPSIS method. In the existing studies, uncertainties, which are present in the data, are handled with either Interval-Valued Intuitionistic Fuzzy Sets (IVIFS) or Intuitionistic Fuzzy Set (IFS) information, which may lose some useful information of alternatives. On the other hand, CIF Set (CIFS) handles the uncertainties by considering both the IVIFS and IFS simultaneously. Thus, motivated by this, in the present work, some series of distance measures between the pairs of CIFSs were presented, and their various relationships were investigated. Further, under this environment, a group decision-making method based on the proposed measure was presented by considering the different priority pairs of the decision-makers. A practical example was provided to verify the developed approach and, demonstrate its practicality and feasibility, their results were compared with those of the several existing approaches.
\end{abstract}

(C) 2020 Sharif University of Technology. All rights reserved.

\section{Introduction}

Multi-Criteria Group Decision-Making (MCGDM) plays a pivotal role in our day-to-day living environment. In this era characterized by cut-throat competition, our target is to select the best alternative from a set of alternatives, to be assessed against the multiple influential criteria. However, selecting only the best alternative does not compile up our problem, but suitable ranking of the all the available options is needed to be done so as to understand their nature and, hence, proceed with further analysis. In such

\footnotetext{
*. Corresponding author.

E-mail addresses: harishg58iitr@gmail.com (H. Garg);

gdeep01@ymail.com (G. Kaur)
}

areas, Decision-Making (DM) approaches act as a boon for the person who has to reach some conclusion by keeping all the favorable and unfavorable conditions in their mind. Traditionally, DM information was assumed to be determinable and clear; however, these properties have not been observed. In practice, due to the increasing complexity of the socioeconomic environment and the problem itself, inaccuracies and cognitive limitations of the human mind can cause decision-makers difficulty in utilizing crisp numbers to express their information $[1,2]$. Thus, the traditional MCGDM method is more limited in real applications. To deal with it, the theory of Fuzzy Set (FS) [3] or extended fuzzy sets, such as Intuitionistic Fuzzy (IF) Set (IFS) [4] and Interval-Valued IFS (IVIFS) [5], are the most successful ones, which characterize the criterion values in terms of membership degrees. Under these environments, various researchers presented 
different kinds of algorithms based on aggregation operator and information measures to solve the MCGDM problems [6-16].

However, apart from that Technique for Order Preference with respect to the Similarity to the Ideal Solution (TOPSIS), developed by Hwang and Yoon [17], there is a well-known Multi-Criteria Decision-Making (MCDM) method. The aim of this method is to choose the best alternative whose distance from its positive ideal solution is the shortest. After their existence, numerous attempts are made by the researchers to apply the TOPSIS method under the fuzzy and IFS environment. For instance, Szmidt and Kacprzyk [18] defined the concept of distance measure between the IFSs. Hung and Yang [19] presented the similarity measures between the two different IFSs based on Hausdorff distance. Boran et al. [20] applied the TOPSIS method to solve the problem of human resource personnel selection. Dugenci [21] presented a distance measure for IVIF set and their application to MCDM with incomplete weight information. Garg [22] presented a generalized improved score function for IVIFSs and their TOPSIS-based method for solving the DM problems. Mohammadi et al. [23] presented a gray relational analysis and TOPSIS approach to solving the DM problems. Garg et al. [24] presented a generalized entropy measure of order $\alpha$ and degree $\beta$ under the IFS environment and applied it to solve the DM problems. Biswas and Kumar [25] presented an integrated TOPSIS approach for solving the DM problems with IVIFS environment. Vommi [26] presented a TOPSIS method using statistical distances to solve DM problems. Singh and Garg [27] developed the distance measures between the type-2 IFS. Li [28] presented a nonlinear programming methodology-based TOPSIS method for solving Multi-Attribute Decision Making (MADM) problems under IVIFS environment. Garg and Arora [29] extended the $\mathrm{Li}$ [28] approach to the interval-valued intuitionistic fuzzy soft set environment. Lu and Ye [30] developed logarithm similarity measures to solve the problems under interval-valued fuzzy set environment. Garg and Kumar [31] presented new similarity measures for IFSs based on the connection number of the set pair analysis. Askarifar et al. [32] presented an approach to studying the framework of Iran's seashores using TOPSIS and best-worst MCDM methods. In [33,34], the authors developed a group DM method under IVIF environment by integrating extended TOPSIS and linear programming methods. Kumar and Garg [35,36] presented the TOPSIS approach for solving DM problems by using connection number of the set pair analysis theory.

Since all these facilitate the uncertainties to a great extent, still they cannot withstand the situations where the decision-maker has to consider the falsity corresponding to the truth value ranging over an interval. However, Cubic Fuzzy Set (CFS) corroborated by Jun et al. [37] is an efficient tool in handling possible disagreement of the agreed interval values, and vice versa. In this set, the degree of agreement/disagreement corresponding to the truth interval has been added to the analysis. Under this environment, Khan et al. [38] and Mahmood et al. [39] presented some aggregation operators under the cubic and cubic-hesitant fuzzy set environments. Fahmi et al. [40] worked on the grey relational analysis method using cubic information and developed an approach to solving the DM problems under CFS environment.

The CFSs take into account only the membership intervals and do not place stress on the nonmembership portion of the data entities. However, in the real world, it is regularly hard to express the estimation of membership degree by an exact value in a FS. In such cases, it might be easier to depict vagueness and uncertainty in the real world using an interval value and an exact value instead of unique interval/exact values. Consequently, the hybrid form of an interval value might be extremely valuable to depict the uncertainties because of his/her reluctant judgment in complex DM problems. For this reason, Kaur and Garg [41,42] introduced the idea of the CIFS, which was described by two parts simultaneously, where one represents the membership degrees by an IVIF Number (IVIFN) and the other represents the membership degrees by IF Number (IFN). Henceforth, a CIFS is the hybrid set joined by both an IVIFN and an IFN. Clearly, the advantage of the CIFS is that it can contain substantially more data to express the IVIFN and IFN at the same time. For instance, suppose that a manager has to evaluate the work of his teammates. The teammate provides him with his self-analyzed report, saying that he has completed 20\%-30\% and simultaneously has not accomplished $50 \%-60 \%$ of the work assigned to him. After analyzing his report by the manager, he passes judgment under IFS environment by saying that he disagrees with the completed work by $20 \%$ and agrees to the incomplete work by $10 \%$. Then, in that case, CIFS is formulated as R-order given by $(\langle[0.20,0.30],[0.50,0.60]\rangle,\langle 0.20,0.10\rangle)$. On the other hand, if the manager agrees by $40 \%$ and disagrees to the incomplete work by $50 \%$, then P-order CIFS is formed as $(\langle[0.20,0.30],[0.50,0.60]\rangle,\langle 0.40,0.50\rangle)$. Therefore, this environment increases the level of precision by enhancing the scope of the membership interval by considering a FS membership value corresponding to it. Hence, it is a useful tool for handling the imprecise and ambiguous information during the DM process under the uncertain environment.

Keeping the advantages of the CIFS, this paper studies the MCGDM problem under CIF setting and proposes a methodology that utilizes extended TOPSIS method where each of the elements is characterized by 
CIF Numbers (CIFNs). CIFNs combine the advantages of both IVIFNs and IFNs. Furthermore, some new weighted and generalized weighted distance measures are proposed in order to signify the level of resemblance between two CIF values based upon the decision values and both the CIF-Positive Ideal Alternative (CIF-PIA) and CIF-Negative Ideal Alternative (CIF-NIA). Several desirable properties of the proposed distance and weighted distance measures are investigated. Multiple decision-makers have been included in the DM process that highlights the impetus of different perspectives, making the proposed approach more realistic for an MCGDM process. The presented approach is illustrated with a numerical example to verify its feasibility and effectiveness. Finally, the computed results obtained by the presented approach are compared with the results of several existing approaches to show the superiority of the former.

The rest of the paper is organized as follows. In Section 2, some basic concepts related to IFSs, IVIFSs, and CIFSs are reviewed. In Section 3, some normalized generalized distance measures are defined for a pair of different CIFNs. Section 4 presents an extended TOPSIS group DM approach for solving the DM problems under the CIFS environment, where each element of the set is characterized by CIFNs. In Section 5, an illustrative example is presented to discuss the functionality of the proposed approach and compare their results with those of some of the existing approaches. Finally, Section 6 summarizes this study.

\section{Preliminaries}

In this section, some basic concepts of IFSs, IVIFSs, $\mathrm{CFSs}$, and CIFSs over the universal set $X$ are presented.

Definition $2.1[4,11]$. An IFS in a set $X$ is an ordered pair defined as follows:

$$
A=\left\{\left(x, \zeta_{A}(x), \vartheta_{A}(x)\right) \mid x \in X\right\},
$$

where $\zeta_{A}$ and $\vartheta_{A}$ are the mappings from $X$ to $[0,1]$, such that $0 \leq \zeta_{A}(x) \leq 1,0 \leq \vartheta_{A}(x) \leq 1$, and $0 \leq$ $\zeta_{A}(x)+\vartheta_{A}(x) \leq 1$ for all $x \in X$. This pair is denoted as $A=\left\langle\zeta_{A}, \vartheta_{A}\right\rangle$ and called as an IFN.

After that, Atanassov and Gargov [5] extend its concept to interval-valued numbers and, hence, defined an IVIFS as follows:

$$
A=\left\{\left\langle x,\left[\zeta_{A}^{L}(x), \zeta_{A}^{U}(x)\right],\left[\vartheta_{A}^{L}(x), \vartheta_{A}^{U}(x)\right]\right\rangle \mid x \in X\right\},
$$

where $0 \leq \zeta_{A}^{L}(x) \leq \zeta_{A}^{U}(x) \leq 1,0 \leq \vartheta_{A}^{L}(x) \leq \vartheta_{A}^{U}(x) \leq$ 1 , and $\zeta_{A}^{U}(x)+\vartheta_{A}^{U}(x) \leq 1$ for all $x$. This pair is often called the IVIFN.

Definition 2.2. Let $A=\left\langle\zeta_{A}, \vartheta_{A}\right\rangle$ and $B=\left\langle\zeta_{B}, \vartheta_{B}\right\rangle$ be two IFNs. Then, the following expressions are defined $[4,11]$ :

(i) $A \subseteq B$ if $\zeta_{A}(x) \leq \zeta_{B}(x)$ and $\vartheta_{A}(x) \geq \vartheta_{B}(x)$ for all $x$ in $X$;

(ii) $A=B$ if and only if $A \subseteq B$ and $B \subseteq A$;

(iii) $A^{c}=\left\{x,\left\langle\vartheta_{A}(x), \zeta_{A}(x)\right\rangle|x \in U\rangle\right\}$;

(iv) $A \cap B=\left\{x,\left\langle\inf \left(\zeta_{A}(x), \zeta_{B}(x)\right), \sup \left(\vartheta_{A}(x), \vartheta_{B}\right.\right.\right.$ $(x))\rangle \mid x \in U\}$

(v) $A \cup B=\left\{x,\left\langle\sup \left(\zeta_{A}(x), \zeta_{B}(x)\right), \inf \left(\vartheta_{A}(x), \vartheta_{B}\right.\right.\right.$ $(x))\rangle \mid x \in U\}$.

Definition 2.3 [37]. A cubic set $\mathcal{A}$ defined in $X$ is given by:

$$
\mathcal{A}=\left\{\left(x, A_{F}(x), \lambda_{F}(x)\right) \mid x \in X\right\},
$$

where $A_{F}(x)=\left[A^{L}(x), A^{U}(x)\right]$ and $\lambda_{F}(x)$ represent the interval-valued FS and FS in $x \in X$, respectively. These pairs are denoted as $\mathcal{A}=\left\langle A_{F}, \lambda_{F}\right\rangle$ and called as cubic fuzzy numbers.

Definition 2.4 [37]. For $\mathcal{A}_{i}=\left\langle A_{i}, \lambda_{i}\right\rangle$ where $i \in \Lambda$, we have:

(i) P-union: $\cup_{i \in \Lambda}^{P} \mathcal{A}_{i}=\left\langle\cup_{i \in \Lambda} A_{i}, \vee_{i \in \Lambda} \lambda_{i}\right\rangle$;

(ii) P-intersection: $\cap_{i \in \Lambda}^{P} \mathcal{A}_{i}=\left\langle\cap_{i \in \Lambda} A_{i}, \wedge_{i \in \Lambda} \lambda_{i}\right\rangle$;

(iii) R-union: $\cup_{i \in \Lambda}^{R} \mathcal{A}_{i}=\left\langle\cup_{i \in \Lambda} A_{i}, \wedge_{i \in \Lambda} \lambda_{i}\right\rangle$;

(iv) R-intersection: $\cap_{i \in \Lambda}^{R} \mathcal{A}_{i}=\left\langle\cap_{i \in \Lambda} A_{i}, \vee_{i \in \Lambda} \lambda_{i}\right\rangle$.

Definition 2.5 $[41,42]$. A CIFS $\mathcal{A}$ defined over the universal set $X$ is an ordered pair, which is defined as follows:

$$
\mathcal{A}=\{\langle x, A(x), \lambda(x)\rangle \mid x \in X\},
$$

where $A=\left\{x,\left\langle\left[\zeta_{A}^{L}(x), \zeta_{A}^{U}(x)\right],\left[\vartheta_{A}^{L}(x), \vartheta_{A}^{U}(x)\right]\right\rangle, \mid x \in\right.$ $X\}$ represents the IVIFS defined on $X$ while:

$$
\lambda(x)=\left\{x,\left\langle\zeta_{A}(x), \vartheta_{A}(x)\right\rangle \mid x \in X\right\}
$$

represents an IFS such that:

$$
\begin{aligned}
& 0 \leq \zeta_{A}^{L}(x) \leq \zeta_{A}^{U}(x) \leq 1, \\
& 0 \leq \vartheta_{A}^{L}(x) \leq \vartheta_{A}^{U}(x) \leq 1, \text { and } \\
& 0 \leq \zeta_{A}^{U}(x)+\vartheta_{A}^{U}(x) \leq 1 .
\end{aligned}
$$

Moreover, $0 \leq \zeta_{A}(x), \vartheta_{A}(x) \leq 1$ and $\zeta_{A}(x)+\vartheta_{A}(x) \leq 1$. For the sake of simplicity, we denote these pairs as $\mathcal{A}=$ $(A, \lambda)$, where $A=\left\langle\left[\zeta_{A}^{L}, \zeta_{A}^{U}\right],\left[\vartheta_{A}^{L}, \vartheta_{A}^{U}\right]\right\rangle$ and $\lambda=\left\langle\zeta_{A}, \vartheta_{A}\right\rangle$ and called as CIFN.

Definition $2.6[41,42]$. Let $\mathcal{A}_{i}=\left(\left\langle\left[\zeta_{i}^{L}, \zeta_{i}^{U}\right],\left[\vartheta_{i}^{L}\right.\right.\right.$, $\left.\left.\left.\vartheta_{i}^{U}\right]\right\rangle,\left\langle\zeta_{i}, \vartheta_{i}\right\rangle\right), i=1,2$ be two CIFNs in $X$. Then, we define: 
(i) (Equality) $\mathcal{A}_{1}=\mathcal{A}_{2} \Leftrightarrow\left[\zeta_{1}^{L}, \zeta_{1}^{U}\right]=\left[\zeta_{2}^{L}, \zeta_{2}^{U}\right]$, $\left[\vartheta_{1}^{L}, \vartheta_{1}^{U}\right]=\left[\vartheta_{2}^{L}, \vartheta_{2}^{U}\right], \zeta_{1}=\zeta_{2}$ and $\vartheta_{1}=\vartheta_{2}$

(ii) (P-order) $\mathcal{A}_{1} \subseteq_{P} \mathcal{A}_{2}$ if $\left[\zeta_{1}^{L}, \zeta_{1}^{U}\right] \subseteq\left[\zeta_{2}^{L}, \zeta_{2}^{U}\right]$, $\left[\vartheta_{1}^{L}, \vartheta_{1}^{U}\right] \supseteq\left[\vartheta_{2}^{L}, \vartheta_{2}^{U}\right], \zeta_{1} \leq \zeta_{2}$ and $\vartheta_{1} \geq \vartheta_{2}$

(iii) (R-order) $\mathcal{A}_{1} \subseteq_{R} \quad \mathcal{A}_{2}$ if $\left[\zeta_{1}^{L}, \zeta_{1}^{U}\right] \subseteq\left[\zeta_{2}^{L}, \zeta_{2}^{U}\right]$, $\left[\vartheta_{1}^{L}, \vartheta_{1}^{U}\right] \supseteq\left[\vartheta_{2}^{L}, \vartheta_{2}^{U}\right], \zeta_{1} \geq \zeta_{2}$ and $\vartheta_{1} \leq \vartheta_{2}$.

\section{Distance measures for CIFS}

In this section, some new distance measures for the non-zero CIFN over the finite universal set $X=$ $\left\{x_{1}, x_{2}, \ldots, x_{n}\right\}$ are proposed. For it, $\phi(X)$ is considered to be a family of CIFSs over the set $X$.

Definition 3.1. A real-valued function $d: \phi(X) \times$ $\phi(X) \rightarrow[0,1]$ is called the distance measure if it satisfies the following properties for $\mathcal{A}, \mathcal{B}, \mathcal{C} \in \phi(X)$ :

(P1) $0 \leq d(\mathcal{A}, \mathcal{B}) \leq 1$

(P2) $d(\mathcal{A}, \mathcal{B})=0$ if and only if $\mathcal{A}=\mathcal{B}$;

(P3) $d(\mathcal{A}, \mathcal{B})=d(\mathcal{B}, \mathcal{A})$;

(P4) If $\mathcal{A} \subseteq \mathcal{B} \subseteq \mathcal{C}$, then $d(\mathcal{A}, \mathcal{B}) \leq d(\mathcal{A}, \mathcal{C})$ and $d(\mathcal{B}, \mathcal{C}) \leq d(\overline{\mathcal{A}}, \mathcal{C})$

where $\phi(\cdot)$ represents the set of all CIFSs.

Definition 3.2. Let $\mathcal{A}=\left(\left\langle\left[\zeta_{A}^{L}(x), \zeta_{A}^{U}(x)\right],\left[\vartheta_{A}^{L}(x)\right.\right.\right.$, $\left.\left.\left.\vartheta_{A}^{U}(x)\right]\right\rangle,\left\langle\left[\zeta_{A}(x), \vartheta_{A}(x)\right]\right\rangle\right)$ and $\mathcal{B}=\left(\left\langle\left[\zeta_{B}^{L}(x), \zeta_{B}^{U}(x)\right]\right.\right.$, $\left.\left.\left[\vartheta_{B}^{L}(x), \vartheta_{B}^{U}(x)\right]\right\rangle,\left\langle\left[\zeta_{B}(x), \vartheta_{B}(x)\right]\right\rangle\right)$ be two CIFNs. Then, for $q \geq 1$, the following distance measures are defined:

(i) Distance measures are defined by Eq. (5) as shown in Box I;

(ii) Normalized distance measures are defined by Eq. (6) as shown in Box II.
Next, we confirm that the above-defined measures are valid distance measures.

Theorem 3.1. The measure, $d_{q}^{\prime}$, between two CIFSs, $\mathcal{A}$ and $\mathcal{B}$, satisfies the properties (P1)-(P4), as defined in Definition 3.1.

Proof. In order to prove that the measure defined in Eq. (5) is a valid distance measure, we shall prove that it satisfies the properties (P1)-(P4), as defined in Definition 3.1, for a collection of CIFNs:

$$
\mathcal{A}=\left(\left\langle\left[\zeta_{A}^{L}(x), \zeta_{A}^{U}(x)\right],\left[\vartheta_{A}^{L}(x), \vartheta_{A}^{U}(x)\right]\right\rangle,\left\langle\left[\zeta_{A}(x), \vartheta_{A}(x)\right]\right\rangle\right),
$$

and:

$$
\mathcal{B}=\left(\left\langle\left[\zeta_{B}^{L}(x), \zeta_{B}^{U}(x)\right],\left[\vartheta_{B}^{L}(x), \vartheta_{B}^{U}(x)\right]\right\rangle,\left\langle\left[\zeta_{B}(x), \vartheta_{B}(x)\right]\right\rangle\right) .
$$

For any real number $q \geq 1$ and a collection of CIFSs $\mathcal{A}$ and $\mathcal{B}$, we have:

(P1) By the definition of $d_{q}^{\prime}$, we have $d_{q}^{\prime}(\mathcal{A}, \mathcal{B}) \geq 0$; thus, for arbitrary CIFSs $\mathcal{A}$ and $\mathcal{B}$, it is enough to show that $d_{q}^{\prime}(\mathcal{A}, \mathcal{B}) \leq 1$. Since $\mathcal{A}$ and $\mathcal{B}$ are two CIFSs, we have:

$$
\begin{aligned}
& 0 \leq \zeta_{A}^{L}\left(x_{i}\right), \zeta_{A}^{U}\left(x_{i}\right), \vartheta_{A}^{L}\left(x_{i}\right), \vartheta_{A}^{U}\left(x_{i}\right) \leq 1, \\
& 0 \leq \zeta_{A}\left(x_{i}\right), \vartheta_{A}\left(x_{i}\right) \leq 1, \\
& 0 \leq \zeta_{B}^{L}\left(x_{i}\right), \zeta_{B}^{U}\left(x_{i}\right), \vartheta_{B}^{L}\left(x_{i}\right), \vartheta_{B}^{U}\left(x_{i}\right) \leq 1, \\
& 0 \leq \zeta_{B}\left(x_{i}\right), \vartheta_{B}\left(x_{i}\right) \leq 1 .
\end{aligned}
$$

This implies that:

$$
\begin{aligned}
& 0 \leq\left|\zeta_{A}^{L}\left(x_{i}\right)-\zeta_{B}^{L}\left(x_{i}\right)\right|^{q} \leq 1, \\
& 0 \leq\left|\zeta_{A}^{U}\left(x_{i}\right)-\zeta_{B}^{U}\left(x_{i}\right)\right|^{q} \leq 1, \\
& 0 \leq\left|\vartheta_{A}^{L}\left(x_{i}\right)-\vartheta_{B}^{L}\left(x_{i}\right)\right|^{q} \leq 1 \\
& 0 \leq\left|\vartheta_{A}^{U}\left(x_{i}\right)-\vartheta_{B}^{U}\left(x_{i}\right)\right|^{q} \leq 1 .
\end{aligned}
$$

$$
d_{q}^{\prime \prime}(\mathcal{A}, \mathcal{B})=\left(\frac{1}{6} \sum_{i=1}^{n}\left\{\begin{array}{l}
\left|\zeta_{A}^{L}\left(x_{i}\right)-\zeta_{B}^{L}\left(x_{i}\right)\right|^{q}+\left|\zeta_{A}^{U}\left(x_{i}\right)-\zeta_{B}^{U}\left(x_{i}\right)\right|^{q}+\left|\vartheta_{A}^{L}\left(x_{i}\right)-\vartheta_{B}^{L}\left(x_{i}\right)\right|^{q} \\
+\left|\vartheta_{A}^{U}\left(x_{i}\right)-\vartheta_{B}^{U}\left(x_{i}\right)\right|^{q}+\left|\zeta_{A}\left(x_{i}\right)-\zeta_{B}\left(x_{i}\right)\right|^{q}+\left|\vartheta_{A}\left(x_{i}\right)-\vartheta_{B}\left(x_{i}\right)\right|^{q}
\end{array}\right\}\right)^{1 / q}
$$

$$
d_{q}^{\prime}(\mathcal{A}, \mathcal{B})=\left(\frac{1}{6 n} \sum_{i=1}^{n}\left\{\begin{array}{l}
\left|\zeta_{A}^{L}\left(x_{i}\right)-\zeta_{B}^{L}\left(x_{i}\right)\right|^{q}+\left|\zeta_{A}^{U}\left(x_{i}\right)-\zeta_{B}^{U}\left(x_{i}\right)\right|^{q}+\left|\vartheta_{A}^{L}\left(x_{i}\right)-\vartheta_{B}^{L}\left(x_{i}\right)\right|^{q} \\
+\left|\vartheta_{A}^{U}\left(x_{i}\right)-\vartheta_{B}^{U}\left(x_{i}\right)\right|^{q}+\left|\zeta_{A}\left(x_{i}\right)-\zeta_{B}\left(x_{i}\right)\right|^{q}+\left|\vartheta_{A}\left(x_{i}\right)-\vartheta_{B}\left(x_{i}\right)\right|^{q}
\end{array}\right\}\right)^{1 / q}
$$


Similarly:

$$
\begin{aligned}
& 0 \leq\left|\zeta_{A}\left(x_{i}\right)-\zeta_{B}\left(x_{i}\right)\right|^{q} \leq 1, \\
& 0 \leq\left|\vartheta_{A}\left(x_{i}\right)-\vartheta_{B}\left(x_{i}\right)\right|^{q} \leq 1 .
\end{aligned}
$$

Thus, it follows that $0 \leq d_{q}^{\prime}(\mathcal{A}, \mathcal{B}) \leq 1$.

(P2) For any two CIFSs $\mathcal{A}$ and $\mathcal{B}$,

$$
\begin{aligned}
& d_{q}^{\prime}(\mathcal{A}, \mathcal{B})=0 \\
\Leftrightarrow & \frac{1}{6 n} \sum_{i=1}^{n}\left\{\left|\zeta_{A}^{L}\left(x_{i}\right)-\zeta_{B}^{L}\left(x_{i}\right)\right|^{q}+\mid \zeta_{A}^{U}\left(x_{i}\right)\right. \\
& -\left.\zeta_{B}^{U}\left(x_{i}\right)\right|^{q}+\left|\vartheta_{A}^{L}\left(x_{i}\right)-\vartheta_{B}^{L}\left(x_{i}\right)\right|^{q}+\mid \vartheta_{A}^{U}\left(x_{i}\right) \\
& -\left.\vartheta_{B}^{U}\left(x_{i}\right)\right|^{q}+\left|\zeta_{A}\left(x_{i}\right)-\zeta_{B}\left(x_{i}\right)\right|^{q}+\mid \vartheta_{A}\left(x_{i}\right) \\
& \left.-\left.\vartheta_{B}\left(x_{i}\right)\right|^{q}\right\}=0 \\
\Leftrightarrow \mid & \zeta_{A}^{L}\left(x_{i}\right)-\left.\zeta_{B}^{L}\left(x_{i}\right)\right|^{q}=0, \mid \zeta_{A}^{U}\left(x_{i}\right) \\
& -\left.\zeta_{B}^{U}\left(x_{i}\right)\right|^{q}=0,\left|\vartheta_{A}^{L}\left(x_{i}\right)-\vartheta_{B}^{L}\left(x_{i}\right)\right|^{q}=0, \\
& \left|\vartheta_{A}^{U}\left(x_{i}\right)-\vartheta_{B}^{U}\left(x_{i}\right)\right|^{q}=0, \mid \zeta_{A}\left(x_{i}\right) \\
& -\left.\zeta_{B}\left(x_{i}\right)\right|^{q}=0 \text { and }\left|\vartheta_{A}\left(x_{i}\right)-\vartheta_{B}\left(x_{i}\right)\right|^{q}=0 ;
\end{aligned}
$$

for all $i$

$$
\begin{gathered}
\Leftrightarrow \zeta_{A}^{L}\left(x_{i}\right)=\zeta_{B}^{L}\left(x_{i}\right), \zeta_{A}^{U}\left(x_{i}\right) \\
=\zeta_{B}^{U}\left(x_{i}\right), \vartheta_{A}^{L}\left(x_{i}\right)=\vartheta_{B}^{L}\left(x_{i}\right), \vartheta_{A}^{U}\left(x_{i}\right)=\vartheta_{B}^{U}\left(x_{i}\right), \\
\zeta_{A}\left(x_{i}\right)=\zeta_{B}\left(x_{i}\right), \text { and } \vartheta_{A}\left(x_{i}\right)=\vartheta_{B}\left(x_{i}\right)
\end{gathered}
$$

for all $i$

$\Leftrightarrow \mathcal{A}=\mathcal{B}$.

(P3) For any two real numbers $a$ and $b$, we have $\mid a-$ $b|=| b-a \mid$. Thus, we have $d_{q}^{\prime}(\mathcal{A}, \mathcal{B})=d_{q}^{\prime}(\mathcal{B}, \mathcal{A})$.

(P4) If $\mathcal{A} \subseteq \mathcal{B} \subseteq \mathcal{C}$ are R-order CIFNs then for all $i$, we have:

$$
\begin{aligned}
{\left[\zeta_{A}^{L}\left(x_{i}\right), \zeta_{A}^{U}\left(x_{i}\right)\right] } & \subseteq\left[\zeta_{B}^{L}\left(x_{i}\right), \zeta_{B}^{U}\left(x_{i}\right)\right] \\
& \subseteq\left[\zeta_{C}^{L}\left(x_{i}\right), \zeta_{C}^{U}\left(x_{i}\right)\right], \\
{\left[\vartheta_{A}^{L}\left(x_{i}\right), \vartheta_{A}^{U}\left(x_{i}\right)\right] } & \supseteq\left[\vartheta_{B}^{L}\left(x_{i}\right), \vartheta_{B}^{U}\left(x_{i}\right)\right] \\
& \supseteq\left[\vartheta_{C}^{L}\left(x_{i}\right), \vartheta_{C}^{U}\left(x_{i}\right)\right], \\
\zeta_{A}\left(x_{i}\right) \geq \zeta_{B}\left(x_{i}\right) & \geq \zeta_{C}\left(x_{i}\right),
\end{aligned}
$$

and:

$$
\vartheta_{A}\left(x_{i}\right) \leq \vartheta_{B}\left(x_{i}\right) \leq \vartheta_{C}\left(x_{i}\right)
$$

Therefore:

$$
\begin{aligned}
& \left|\zeta_{A}^{L}\left(x_{i}\right)-\zeta_{B}^{L}\left(x_{i}\right)\right|^{q} \leq\left|\zeta_{A}^{L}\left(x_{i}\right)-\zeta_{C}^{L}\left(x_{i}\right)\right|^{q}, \\
& \left|\zeta_{A}^{U}\left(x_{i}\right)-\zeta_{B}^{U}\left(x_{i}\right)\right|^{q} \leq\left|\zeta_{A}^{U}\left(x_{i}\right)-\zeta_{C}^{U}\left(x_{i}\right)\right|^{q}, \\
& \left|\vartheta_{A}^{L}\left(x_{i}\right)-\vartheta_{B}^{L}\left(x_{i}\right)\right|^{q} \geq\left|\vartheta_{A}^{L}\left(x_{i}\right)-\vartheta_{C}^{L}\left(x_{i}\right)\right|^{q}, \\
& \left|\vartheta_{A}^{U}\left(x_{i}\right)-\vartheta_{B}^{U}\left(x_{i}\right)\right|^{q} \geq\left|\vartheta_{A}^{U}\left(x_{i}\right)-\vartheta_{C}^{U}\left(x_{i}\right)\right|^{q}, \\
& \left|\zeta_{A}\left(x_{i}\right)-\zeta_{B}\left(x_{i}\right)\right|^{q} \geq\left|\zeta_{A}\left(x_{i}\right)-\zeta_{C}\left(x_{i}\right)\right|^{q}, \\
& \left|\vartheta_{A}\left(x_{i}\right)-\vartheta_{B}\left(x_{i}\right)\right|^{q} \leq\left|\vartheta_{A}\left(x_{i}\right)-\vartheta_{C}\left(x_{i}\right)\right|^{q} .
\end{aligned}
$$

Thus:

$$
\begin{aligned}
d_{q}^{\prime}(\mathcal{A}, \mathcal{C}) & =\left[\frac { 1 } { 6 n } \sum _ { i = 1 } ^ { n } \left\{\left|\zeta_{A}^{L}\left(x_{i}\right)-\zeta_{C}^{L}\left(x_{i}\right)\right|^{q}\right.\right. \\
& +\left|\zeta_{A}^{U}\left(x_{i}\right)-\zeta_{C}^{U}\left(x_{i}\right)\right|^{q}+\left|\vartheta_{A}^{L}\left(x_{i}\right)-\vartheta_{C}^{L}\left(x_{i}\right)\right|^{q} \\
& +\left|\vartheta_{A}^{U}\left(x_{i}\right)-\vartheta_{C}^{U}\left(x_{i}\right)\right|^{q}+\left|\zeta_{A}\left(x_{i}\right)-\zeta_{C}\left(x_{i}\right)\right|^{q} \\
& \left.\left.+\left|\vartheta_{A}\left(x_{i}\right)-\vartheta_{C}\left(x_{i}\right)\right|^{q}\right\}\right]^{1 / q} \\
& \geq\left[\frac { 1 } { 6 n } \sum _ { i = 1 } ^ { n } \left\{\left|\zeta_{A}^{L}\left(x_{i}\right)-\zeta_{B}^{L}\left(x_{i}\right)\right|^{q}\right.\right. \\
& +\left|\zeta_{A}^{U}\left(x_{i}\right)-\zeta_{B}^{U}\left(x_{i}\right)\right|^{q}+\left|\vartheta_{A}^{L}\left(x_{i}\right)-\vartheta_{B}^{L}\left(x_{i}\right)\right|^{q} \\
& +\left|\vartheta_{A}^{U}\left(x_{i}\right)-\vartheta_{B}^{U}\left(x_{i}\right)\right|^{q}+\left|\zeta_{A}\left(x_{i}\right)-\zeta_{B}\left(x_{i}\right)\right|^{q} \\
& \left.\left.+\left|\vartheta_{A}\left(x_{i}\right)-\vartheta_{B}\left(x_{i}\right)\right|^{q}\right\}\right]^{1 / q} .
\end{aligned}
$$

Hence, $d_{q}^{\prime}(\mathcal{A}, \mathcal{C}) \geq d_{q}^{\prime}(\mathcal{A}, \mathcal{B})$. Thus, $d_{q}^{\prime}(\mathcal{A}, \mathcal{C}) \geq$ $d_{q}^{\prime}(\mathcal{B}, \mathcal{C})$. Similarly, we can prove it for $\mathrm{P}$-order CIFNs.

Hence, $d_{q}^{\prime}(q \geq 1)$ is a valid distance measure.

Theorem 3.2. The measure, $d_{q}^{\prime \prime}$, satisfies the inequality $d_{q}^{\prime \prime} \leq n^{1 / q}$.

Proof. For any real number $q \geq 1$ and for two CIFSs, $\mathcal{A}$ and $\mathcal{B}$, we have $\left|\zeta_{A}^{L}\left(x_{i}\right)-\zeta_{B}^{L}\left(x_{i}\right)\right|^{q} \leq 1, \mid \zeta_{A}^{U}\left(x_{i}\right)-$ $\left.\zeta_{B}^{U}\left(x_{i}\right)\right|^{q} \leq 1$, and so on. Therefore, we get: 


$$
\begin{aligned}
d_{q}^{\prime \prime}(\mathcal{A}, \mathcal{B}) & =\left(\frac { 1 } { 6 } \sum _ { i = 1 } ^ { n } \left\{\left|\zeta_{A}^{L}\left(x_{i}\right)-\zeta_{B}^{L}\left(x_{i}\right)\right|^{q}\right.\right. \\
& +\left|\zeta_{A}^{U}\left(x_{i}\right)-\zeta_{B}^{U}\left(x_{i}\right)\right|^{q}+\left|\vartheta_{A}^{L}\left(x_{i}\right)-\vartheta_{B}^{L}\left(x_{i}\right)\right|^{q} \\
& +\left|\vartheta_{A}^{U}\left(x_{i}\right)-\vartheta_{B}^{U}\left(x_{i}\right)\right|^{q}+\left|\zeta_{A}\left(x_{i}\right)-\zeta_{B}\left(x_{i}\right)\right|^{q} \\
& \left.\left.+\left|\vartheta_{A}\left(x_{i}\right)-\vartheta_{B}\left(x_{i}\right)\right|^{q}\right\}\right)^{1 / q} \\
& \leq\left(\frac{1}{6} \sum_{i=1}^{n}(1+1+1+1+1+1)\right)^{1 / q} \\
& \leq n^{1 / q}
\end{aligned}
$$

Hence, the result is found.

Theorem 3.3. The measures $d_{q}^{\prime \prime}$ and $d_{q}^{\prime}$ satisfy the inequality $d_{q}^{\prime} \leq \sqrt[q]{d_{1}^{\prime}}$ and $d_{q}^{\prime \prime} \leq \sqrt[q]{d_{1}^{\prime \prime}}$.

Proof. For any real number $q \geq 1$ and for two CIFSs $\mathcal{A}$ and $\mathcal{B}$, we have $\left|\zeta_{A}^{L}\left(x_{i}\right)-\zeta_{B}^{L}\left(x_{i}\right)\right|^{q} \leq\left|\zeta_{A}^{L}\left(x_{i}\right)-\zeta_{B}^{L}\left(x_{i}\right)\right|$, $\left|\zeta_{A}^{U}\left(x_{i}\right)-\zeta_{B}^{U}\left(x_{i}\right)\right|^{q} \leq\left|\zeta_{A}^{U}\left(x_{i}\right)-\zeta_{B}^{U}\left(x_{i}\right)\right|$, and so on. Therefore, we get:

$$
\begin{aligned}
d_{q}^{\prime}(\mathcal{A}, \mathcal{B})= & \left(\frac { 1 } { 6 n } \sum _ { i = 1 } ^ { n } \left\{\left|\zeta_{A}^{L}\left(x_{i}\right)-\zeta_{B}^{L}\left(x_{i}\right)\right|^{q}\right.\right. \\
& +\left|\zeta_{A}^{U}\left(x_{i}\right)-\zeta_{B}^{U}\left(x_{i}\right)\right|^{q}+\left|\vartheta_{A}^{L}\left(x_{i}\right)-\vartheta_{B}^{L}\left(x_{i}\right)\right|^{q} \\
& +\left|\vartheta_{A}^{U}\left(x_{i}\right)-\vartheta_{B}^{U}\left(x_{i}\right)\right|^{q}+\left|\zeta_{A}\left(x_{i}\right)-\zeta_{B}\left(x_{i}\right)\right|^{q} \\
& \left.\left.+\left|\vartheta_{A}\left(x_{i}\right)-\vartheta_{B}\left(x_{i}\right)\right|^{q}\right\}\right)^{1 / q} \\
& \leq\left(\frac { 1 } { 6 n } \sum _ { i = 1 } ^ { n } \left\{\left|\zeta_{A}^{L}\left(x_{i}\right)-\zeta_{B}^{L}\left(x_{i}\right)\right|\right.\right. \\
& +\left|\zeta_{A}^{U}\left(x_{i}\right)-\zeta_{B}^{U}\left(x_{i}\right)\right|+\left|\vartheta_{A}^{L}\left(x_{i}\right)-\vartheta_{B}^{L}\left(x_{i}\right)\right| \\
& +\left|\vartheta_{A}^{U}\left(x_{i}\right)-\vartheta_{B}^{U}\left(x_{i}\right)\right|+\left|\zeta_{A}\left(x_{i}\right)-\zeta_{B}\left(x_{i}\right)\right| \\
& \left.\left.+\left|\vartheta_{A}\left(x_{i}\right)-\vartheta_{B}\left(x_{i}\right)\right|\right\}\right)^{1 / q} \leq\left(d_{1}^{\prime}(\mathcal{A}, \mathcal{B})\right)^{1 / q}
\end{aligned}
$$

Similarly, we can prove that $d_{q}^{\prime \prime} \leq \sqrt[q]{d_{1}^{\prime \prime}}$.

Theorem 3.4. The measures $d_{q}^{\prime \prime}$ and $d_{q}^{\prime}$ satisfy the equation $d_{q}^{\prime \prime}=n^{1 / q} d_{q}^{\prime}$.

Proof. They easily follow from the definitions of $d_{q}^{\prime}$ and $d_{q}^{\prime \prime}$.

Remark 3.1. From the proposed measure, it has been observed that:

(i) When $q=1$, Eq. (6) reduces to the normalized hamming distance measure, and

(ii) When $q=2$, Eq. (6) reduces to the normalized Euclidean distance measure.

As in practical situations, many times, we have to deal with such situations in which various CIFSs may have weights assigned to them. Therefore, taking weights $\omega_{i}(i=1,2, \ldots, n)$ into account, where each $\omega_{i}>0$ and $\sum_{i=1}^{n} \omega_{i}=1$, the generalized weighted distances between two CIFSs $\mathcal{A}$ and $\mathcal{B}$ are defined by Eq. (7) as shown in Box III.

Theorem 3.5. The weighted distance measure $d_{q}$, $(1 \leq q<\infty)$, defined in Eq. (7), satisfies the following properties:

(P1) $0 \leq d_{q}(\mathcal{A}, \mathcal{B}) \leq 1$;

(P2) $d_{q}(\mathcal{A}, \mathcal{B})=0 \Leftrightarrow \mathcal{A}=\mathcal{B}$

(P3) $d_{q}(\mathcal{A}, \mathcal{B})=d_{q}(\mathcal{B}, \mathcal{A})$;

(P4) If $\mathcal{A} \subseteq \mathcal{B} \subseteq \mathcal{C}$ then $d_{q}(\mathcal{A}, \mathcal{B}) \leq d_{q}(\mathcal{A}, \mathcal{C})$ and $d_{q}(\mathcal{B}, \mathcal{C}) \leq d_{q}(\mathcal{A}, \mathcal{C})$.

Proof. The proof is similar to Theorem 3.1; hence, it is omitted here.

Theorem 3.6. The measures $d_{q}^{\prime}, d_{q}^{\prime \prime}$, and $d_{q}$ satisfy the following inequalities:

(i) $d_{q}^{\prime} \leq d_{q}^{\prime \prime} \leq \sqrt[q]{d_{1}^{\prime \prime}}$;

(ii) $d_{q} \leq d_{q}^{\prime \prime} \leq \sqrt[q]{d_{1}^{\prime \prime}}$.

Proof. Since $\omega_{i}>0$ and $\sum_{i=1}^{n} \omega_{i}=1$, we follow the results from their definitions.

Remark 3.2. From this weighted measure, it has been observed that:

$$
d_{q}(\mathcal{A}, \mathcal{B})=\left(\frac{1}{6} \sum_{i=1}^{n} \omega_{i}\left\{\begin{array}{l}
\left|\zeta_{A}^{L}\left(x_{i}\right)-\zeta_{B}^{L}\left(x_{i}\right)\right|^{q}+\left|\zeta_{A}^{U}\left(x_{i}\right)-\zeta_{B}^{U}\left(x_{i}\right)\right|^{q}+\left|\vartheta_{A}^{L}\left(x_{i}\right)-\vartheta_{B}^{L}\left(x_{i}\right)\right|^{q} \\
+\left|\vartheta_{A}^{U}\left(x_{i}\right)-\vartheta_{B}^{U}\left(x_{i}\right)\right|^{q}+\left|\zeta_{A}\left(x_{i}\right)-\zeta_{B}\left(x_{i}\right)\right|^{q}+\left|\vartheta_{A}\left(x_{i}\right)-\vartheta_{B}\left(x_{i}\right)\right|^{q}
\end{array}\right\}\right)^{1 / q}
$$


(i) If $q=1$, then Eq. (7) reduces to the weighted Hamming distance;

(ii) If $q=2$, then Eq. (7) is called the weighted Euclidean distance;

(iii) In particular, when $\omega_{i}=1 / n$, for $i=1,2, \ldots, n$, then Eq. (7) reduces to Eq. (6).

\section{An extended TOPSIS approach based on the proposed distance}

In this section, a TOPSIS approach is presented under the CIFNs environment for solving MCGDM problems based on the proposed distance measure.

\subsection{Description of the problem}

Assume that there is a set of $m$ alternatives, $A=$ $\left\{A_{1}, A_{2}, \ldots, A_{m}\right\}$, that are evaluated under the set of $n$ different criteria, $C=\left\{C_{1}, C_{2}, \ldots, C_{n}\right\}$, such that their rating values are summarized in the form of CIFNs $\alpha_{i j}=\left(A_{i j}, \lambda_{i j}\right)$, where $A_{i j}=\left\langle\left[\zeta_{i j}^{L}, \zeta_{i j}^{U}\right], \quad\left[\vartheta_{i j}^{L}, \vartheta_{i j}^{U}\right]\right\rangle$ represent the IVIFNs and $\lambda_{i j}=\left\langle\zeta_{i j}, \vartheta_{i j}\right\rangle$ represent the IFNs. Here, the components $\left[\zeta_{i j}^{L}, \zeta_{i j}^{U}\right]$ and $\vartheta_{i j}$ represent the degree up to which the given alternative $A_{i}$ satisfies the criterion $C_{j}$, whereas the components $\left[\vartheta_{i j}^{L}, \vartheta_{i j}^{U}\right]$ and $\zeta_{i j}$ indicate the dissatisfaction degree of alternative $A_{i}$ regarding the criterion $C_{j}$. Thus, the overall representation of these rating values can be framed into the CIFN environment and, hence, the collective decision matrix is summarized as $D=\left(\alpha_{i j}\right)_{m \times n}$.

\subsection{Computation of CIF-PIA and CIF-NIA}

Since all of the rating values of the alternatives are CIFNs, the CIF-PIA; and CIF-NIA on the alternative $A_{i} \quad(i=1,2, \ldots, m)$ may be chosen as 1 , and 0 respectively. Thus, rating values of CIF-PIA and CIFNIA are expressed as follows:

$$
\alpha^{+}=(\langle[1,1],[0,0]\rangle,\langle 0,1\rangle)_{1 \times n},
$$

and:

$$
\alpha^{-}=(\langle[0,0],[1,1]\rangle,\langle 1,0\rangle)_{1 \times n} .
$$

From these, it has been seen that $\alpha^{+}$and $\alpha^{-}$complement each other.
However, if we take the fixed a priori CIFPIA and CIF-NIA reference points, then the overall performance value and, hence, the ranking order of the alternatives could not change if the alternatives are changed. Instead, the decision-maker wants to define these reference points as follows:

$$
\alpha_{j}^{+}=\left(\left\langle\left[g_{j}^{L+}, g_{j}^{U+}\right],\left[h_{j}^{L+}, h_{j}^{U+}\right]\right\rangle,\left\langle r_{j}^{+}, s_{j}^{+}\right\rangle\right),
$$

and:

$$
\alpha_{j}^{-}=\left(\left\langle\left[g_{j}^{L-}, g_{j}^{U-}\right],\left[h_{j}^{L-}, h_{j}^{U-}\right]\right\rangle,\left\langle r_{j}^{-}, s_{j}^{-}\right\rangle\right),
$$

where:

$$
\begin{array}{ll}
g_{j}^{L+}=\max _{j}\left\{\zeta_{i j}^{L}\right\}, & \left.g_{j}^{U+}=\max _{j}\left\{\zeta_{i j}^{U}\right)\right\}, \\
\left.h_{j}^{L+}=\min _{j}\left\{\vartheta_{i j}^{L}\right)\right\}, & \left.h_{j}^{U+}=\min _{j}\left\{\vartheta_{i j}^{U}\right)\right\}, \\
\left.r_{j}^{+}=\min _{j}\left\{\zeta_{i j}\right)\right\}, & \left.s_{j}^{+}=\max _{j}\left\{\vartheta_{i j}\right)\right\}, \\
\left.g_{j}^{L-}=\min _{j}\left\{\zeta_{i j}^{L}\right)\right\}, & \left.g_{j}^{U-}=\min _{j}\left\{\zeta_{i j}^{U}\right)\right\}, \\
\left.h_{j}^{L-}=\max _{j}\left\{\vartheta_{i j}^{L}\right)\right\}, & \left.h_{j}^{U-}=\max _{j}\left\{\vartheta_{i j}^{U}\right)\right\}, \\
\left.r_{j}^{-}=\max _{j}\left\{\zeta_{i j}\right)\right\}, & \left.s_{j}^{-}=\min _{j}\left\{\vartheta_{i j}\right)\right\},
\end{array}
$$

for all $i$.

\subsection{Computation of distance measures between the alternatives}

By considering the importance of the criteria in terms of weight vector $\omega=\left(\omega_{1}, \omega_{2}, \ldots, \omega_{n}\right)^{T}$ along with CIFPIA $\left(\alpha^{+}\right)$and CIF-NIA $\left(\alpha^{-}\right)$, the weighted distances between the alternatives $A_{i}$ and $\alpha^{+}$, as well as $\alpha^{-}$, are computed by Eqs. (10) and (11) as shown in Box IV; $q \geq 1$ is a real number.

Based on these weighted distances, the relative closeness coefficient of alternative $A_{i}(i=1,2, \ldots, n)$ with respect to CIF-PIA $\alpha^{+}$is given as follows:

$$
\mathfrak{C}_{i}=\frac{d_{q}\left(A_{i}, \alpha^{-}\right)}{d_{q}\left(A_{i}, \alpha^{-}\right)+d_{q}\left(A_{i}, \alpha^{+}\right)} ; \quad d_{q}\left(A_{i}, \alpha^{+}\right) \neq 0 .
$$

Further, it has been seen that, $0 \leq d_{q}\left(A_{i}, \alpha^{-}\right) \leq$ $d_{q}\left(A_{i}, \alpha^{-}\right)+d_{q}\left(A_{i}, \alpha^{+}\right)$and hence $0 \leq \mathfrak{C}_{i} \leq 1$.

$$
\begin{aligned}
& d_{q}\left(A_{i}, \alpha^{+}\right)=\left(\frac{1}{6} \sum_{j=1}^{n} \omega_{j}\left\{\begin{array}{l}
\left|g_{j}^{L+}-\zeta_{i j}^{L}\right|^{q}+\left|g_{j}^{U+}-\zeta_{i j}^{U}\right|^{q}+\left|\vartheta_{i j}^{L}-h_{j}^{L+}\right|^{q} \\
+\left|\vartheta_{i j}^{U}-h_{j}^{U+}\right|^{q}+\left|\zeta_{i j}-r_{j}^{+}\right|^{q}+\left|s_{j}^{+}-\vartheta_{i j}\right|^{q}
\end{array}\right\}\right)^{\frac{1}{q}}, \\
& d_{q}\left(A_{i}, \alpha^{-}\right)=\left(\frac{1}{6} \sum_{j=1}^{n} \omega_{j}\left\{\begin{array}{l}
\left|\zeta_{i j}^{L}-g_{j}^{L-}\right|^{q}+\left|\zeta_{i j}^{U}-g_{j}^{U-}\right|^{q}+\left|h_{j}^{L+}-\vartheta_{i j}^{L}\right|^{q} \\
+\left|h_{j}^{U+}-\vartheta_{i j}^{U}\right|^{q}+\left|r_{j}^{-}-\zeta_{i j}\right|^{q}+\left|\vartheta_{i j}-s_{j}^{-}\right|^{q}
\end{array}\right\}\right)^{\frac{1}{q}} .
\end{aligned}
$$




\subsection{The proposed group DM TOPSIS approach}

Based on the above analysis, an approach for solving the group DM problems under the CIFN environment has been presented. In doing so, consider that there are ' $K$ ' decision makers $\left\{D^{(1)}, D^{(2)}, \ldots, D^{(K)}\right\}$ which are evaluating the given set of ' $m$ ' alternatives $A_{i}(i=1,2, \ldots, m)$ under the set of ' $n$ ' criteria $C_{j}(j=1,2, \ldots, n)$. These decision makers give their preferences in terms of:

$$
\begin{aligned}
\operatorname{CIFNs}\left(\alpha_{i j}\right)^{(k)} & =\left(\left\langle\left[\left(\zeta_{i j}^{L}\right)^{(k)},\left(\zeta_{i j}^{U}\right)^{(k)}\right],\right.\right. \\
& {\left.\left.\left[\left(\vartheta_{i j}^{L}\right)^{(k)},\left(\vartheta_{i j}^{U}\right)^{(k)}\right]\right\rangle,\left\langle\left(\zeta_{i j}\right)^{(k)},\left(\vartheta_{i j}\right)^{(k)}\right\rangle\right), }
\end{aligned}
$$

where $k=1,2, \ldots, K$. Further, assume that $\omega^{(k)}=$ $\left(\omega_{1}^{(k)}, \omega_{2}^{(k)}, \ldots, \omega_{n}^{(k)}\right)^{T}$ such that each $\omega_{j}^{(k)}>0$ and $\sum_{j=1}^{n} \omega_{j}^{(k)}=1$ be the weight vectors of the criteria. Moreover, in order to overcome the diverse judgements by different experts, their opinion is prioritized in accordance with the weight vector $\lambda=\left(\lambda_{1}, \lambda_{2}, \ldots, \lambda_{K}\right)$ such that $\lambda_{k}>0$ and $\sum_{k=1}^{K} \lambda_{k}=1$. Then, the following steps of the proposed approach are summarized as follows:

Step 1: Arrange the rating values of the alternative given by each decision-maker in the matrix form as follows:

$$
\begin{aligned}
& \begin{array}{llll}
C_{1} & C_{2} & \ldots & C_{n}
\end{array} \\
& D^{(k)}=\begin{array}{c}
A_{1} \\
A_{2} \\
\vdots \\
A_{m}
\end{array}\left(\begin{array}{cccc}
\alpha_{11}^{(k)} & \alpha_{12}^{(k)} & \ldots & \alpha_{1 n}^{(k)} \\
\alpha_{21}^{(k)} & \alpha_{22}^{(k)} & \ldots & \alpha_{2 n}^{(k)} \\
\vdots & \vdots & \ddots & \vdots \\
\alpha_{m 1}^{(k)} & \alpha_{m 2}^{(k)} & \ldots & \alpha_{m n}^{(k)}
\end{array}\right)
\end{aligned}
$$

Step 2: For each decision maker $D^{(k)}, k=$ $1,2, \ldots, K$, compute CIF-PIA and CIF-NIA corresponding to alternative $A_{i} ; i=1,2, \ldots, m$ by using Eqs. (8) and (9), respectively, and are defined as follows:

$$
\begin{aligned}
\left(\alpha^{+}\right)^{(k)}= & \left(\left\langle\left[\left(g_{j}^{L+}\right)^{(k)},\left(g_{j}^{U+}\right)^{(k)}\right],\left[\left(h_{j}^{L+}\right)^{(k)},\left(h_{j}^{U+}\right)^{(k)}\right]\right\rangle,\right. \\
& \left.\left\langle\left[\left(r_{j}^{+}\right)^{(k)},\left(s_{j}^{+}\right)^{(k)}\right]\right\rangle\right),
\end{aligned}
$$

and:

$$
\begin{aligned}
\left(\alpha^{-}\right)^{(k)}= & \left\langle\left\langle\left[\left(g_{j}^{L-}\right)^{(k)},\left(g_{j}^{U-}\right)^{(k)}\right],\left[\left(h_{j}^{L-}\right)^{(k)},\left(h_{j}^{U-}\right)^{(k)}\right]\right\rangle\right. \\
& \left.\left\langle\left[\left(r_{j}^{-}\right)^{(k)},\left(s_{j}^{-}\right)^{(k)}\right]\right\rangle\right)
\end{aligned}
$$

where:

$$
\begin{aligned}
& \left(g_{j}^{L+}\right)^{(k)}=\max _{j}\left\{\left(\zeta_{i j}^{L}\right)^{(k)}\right\}, \\
& \left(g_{j}^{U+}\right)^{(k)}=\max _{j}\left\{\left(\zeta_{i j}^{U}\right)^{(k)}\right\}, \\
& \left(h_{j}^{L+}\right)^{(k)}=\min _{j}\left\{\left(\vartheta_{i j}^{L}\right)^{(k)}\right\}, \\
& \left(h_{j}^{U+}\right)^{(k)}=\min _{j}\left\{\left(\vartheta_{i j}^{U}\right)^{(k)}\right\}, \\
& \left(r_{j}^{+}\right)^{(k)}=\min _{j}\left\{\left(\zeta_{i j}\right)^{(k)}\right\}, \\
& \left(s_{j}^{+}\right)^{(k)}=\max _{j}\left\{\left(\vartheta_{i j}\right)^{(k)}\right\}, \\
& \left(g_{j}^{L-}\right)^{(k)}=\min _{j}\left\{\left(\zeta_{i j}^{L}\right)^{(k)}\right\}, \\
& \left(g_{j}^{U-}\right)^{(k)}=\min _{j}\left\{\left(\zeta_{i j}^{U}\right)^{(k)}\right\}, \\
& \left(h_{j}^{L-}\right)^{(k)}=\max _{j}\left\{\left(\vartheta_{i j}^{L}\right)^{(k)}\right\}, \\
& \left(h_{j}^{U-}\right)^{(k)}=\max _{j}\left\{\left(\vartheta_{i j}^{U}\right)^{(k)}\right\}, \\
& \left(r_{j}^{-}\right)^{(k)}=\max _{j}\left\{\left(\zeta_{i j}\right)^{(k)}\right\}, \\
& \left(s_{j}^{-}\right)^{(k)}=\min _{j}\left\{\left(\vartheta_{i j}\right)^{(k)}\right\} .
\end{aligned}
$$

Step 3: For each decision maker, compute the separation measures between the alternatives $A_{i}$ from its CIF-PIA and CIF-NIA, denoted by $d_{q}\left(\left(A_{i}\right)^{(k)},\left(\alpha^{+}\right)^{(k)}\right)$ and $d_{q}\left(\left(A_{i}\right)^{(k)},\left(\alpha^{-}\right)^{(k)}\right)$, respectively;

Step 4: For each decision-maker, the relative closeness coefficient is determined as follows:

$$
\begin{aligned}
& \mathfrak{C}_{i}^{(k)}=\frac{d_{q}\left(\left(A_{i}\right)^{(k)},\left(\alpha^{-}\right)^{(k)}\right)}{d_{q}\left(\left(A_{i}\right)^{(k)},\left(\alpha^{+}\right)^{(k)}\right)+d_{q}\left(\left(A_{i}\right)^{(k)},\left(\alpha^{-}\right)^{(k)}\right)}, \\
& k=1,2, \ldots, K .
\end{aligned}
$$

where $d_{q}\left(\left(A_{i}\right)^{(k)},\left(\alpha^{+}\right)^{(k)}\right) \neq 0$.

Step 5: Since each decision-maker may have obtained different rankings towards the alternatives, the overall finding of the best alternative remains unclear. In order to overcome these variable rankings, different values of the experts are aggregated by assigning a priority value, $\lambda=\left(\lambda_{1}, \lambda_{2}, \ldots, \lambda_{K}\right)^{T}$ to each expert such that $\lambda_{k}>0$ and $\sum_{k=1}^{K} \lambda_{k}=1$. The separation measures of each expert are aggre- 
gated by using these weight vectors, and the overall measurement values of the alternative are obtained as follows:

$$
\begin{aligned}
& D_{i}^{+}=\sum_{k=1}^{K} \lambda_{k} d_{q}\left(\left(A_{i}\right)^{(k)},\left(\alpha^{+}\right)^{(k)}\right), \\
& D_{i}^{-}=\sum_{k=1}^{K} \lambda_{k} d_{q}\left(\left(A_{i}\right)^{(k)},\left(\alpha^{-}\right)^{(k)}\right) .
\end{aligned}
$$

Step 6: Based on these values, $D_{i}^{+}$and $D_{i}^{-}$, the closeness coefficient for an alternative $A_{i}(i=$ $1,2, \ldots, m)$ is determined as follows:

$$
\mathfrak{C}_{i}=\frac{D_{i}^{-}}{D_{i}^{+}+D_{i}^{-}} ; \quad D_{i}^{+} \neq 0 .
$$

Step 7: Rank the alternative(s) based on the descending values of $\mathfrak{C}_{i}$ 's.

\section{Illustrative example}

In order to demonstrate the above-mentioned approach, an illustrative example is taken as follows.

\subsection{Case study}

A multinational company has started its recruitment process to select the best candidate for the new project.
To do so, a company has published a notification in the newspaper and, based on it, different candidates have applied for it. Then, four candidates $A_{i} ; i=1,2,3,4$ are to be selected for the interview. To evaluate the candidates, the company manager has invited four decision-makers $D^{(1)}, D^{(2)}, D^{(3)}$, and $D^{(4)}$ and given them responsibilities to find the best candidate for the company. The panel has decided to evaluate the candidates $A_{i} ; i=1,2,3,4$ on the basis of four criteria namely $C_{1}$ : 'Educational qualification'; $C_{2}$ : 'Technical knowledge'; $C_{3}$ : 'Communication skills'; $C_{4}$ : 'Work experience'. In doing so, they firstly conducted Group Discussions (GDs) with all the candidates and the results for each candidate are formulated by a panel in the form of IVIFNs. Among the pool of applicants appearing for GD, four candidates were shortlisted for personal interview, and the results for this stage of the recruitment process are recorded in the form of IFNs. Then, the following steps of the proposed approach are executed in order to find the best candidate(s) for the required post:

Step 1: The rating values of each decision-maker in the evaluation of the given alternatives are summarized in Table 1. In this table, rating values under both the recruitment stages are clubbed, which are

\begin{tabular}{|c|c|c|c|c|c|}
\hline 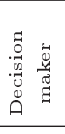 & 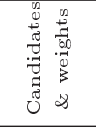 & $C_{1}$ & $C_{2}$ & $C_{3}$ & $C_{4}$ \\
\hline \multirow{5}{*}{$D^{(1)}$} & $A_{1}$ & $(\langle[0.15,0.30],[0.35,0.40]\rangle,\langle 0.20,0.65\rangle)$ & $(\langle[0.13,0.25],[0.40,0.45]\rangle,\langle 0.30,0.60\rangle)$ & $(\langle[0.30,0.45],[0.25,0.30]\rangle,\langle 0.55,0.33\rangle)$ & $(\langle[0.10,0.30],[0.25,0.35]\rangle,\langle 0.11,0.20\rangle)$ \\
\hline & $A_{2} \quad($ & $(\langle[0.10,0.15],[0.35,0.40]\rangle,\langle 0.40,0.17\rangle)$ & $(\langle[0.15,0.22],[0.27,0.30]\rangle,\langle 0.15,0.29\rangle)$ & $(\langle[0.40,0.45],[0.21,0.33]\rangle,\langle 0.16,0.35\rangle)$ & $(\langle[0.50,0.60],[0.15,0.20]\rangle,\langle 0.35,0.19\rangle)$ \\
\hline & $A_{3}$ & $(\langle[0.14,0.25],[0.35,0.65]\rangle,\langle 0.10,0.40\rangle)$ & $(\langle[0.35,0.45],[0.15,0.20]\rangle,\langle 0.30,0.50\rangle)$ & $(\langle[0.45,0.55],[0.15,0.25]\rangle,\langle 0.20,0.80\rangle)$ & $(\langle[0.30,0.50],[0.10,0.30]\rangle,\langle 0.20,0.35\rangle)$ \\
\hline & $A_{4}$ & $(\langle[0.30,0.35],[0.25,0.45]\rangle,\langle 0.20, .30\rangle)$ & $(\langle[0.20,0.55],[0.40,0.45]\rangle,\langle 0.20,0.45\rangle)$ & $(\langle[0.15,0.25],[0.20,0.35]\rangle,\langle 0.60,0.20\rangle)$ & $(\langle[0.10,0.29],[0.40,0.50]\rangle,\langle 0.30,0.40\rangle)$ \\
\hline & Weights & 0.17 & 0.30 & 0.13 & 0.40 \\
\hline \multirow{5}{*}{$D^{(2)}$} & $A_{1}$ & $(\langle[0.10,0.30],[0.35,0.45]\rangle,\langle 0.60,0.10\rangle)$ & $(\langle[0.15,0.20],[0.25,0.29]\rangle,\langle 0.18,0.66\rangle)$ & $(\langle[0.44,0.50],[0.20,0.30]\rangle,\langle 0.18,0.35\rangle)$ & $(\langle[0.10,0.30],[0.25,0.35]\rangle,\langle 0.11,0.20\rangle)$ \\
\hline & $A_{2}$ & $(\langle[0.20,0.30],[0.40,0.50]\rangle,\langle 0.10,0.40\rangle)$ & $(\langle[0.30,0.40],[0.10,0.60]\rangle,\langle 0.20,0.40\rangle)$ & $(\langle[0.40,0.50],[0.20,0.30]\rangle,\langle 0.60,0.30\rangle)$ & $(\langle[0.10,0.50],[0.20,0.30]\rangle,\langle 0.40,0.30\rangle)$ \\
\hline & $A_{3}$ & $(\langle[0.10,0.20],[0.30,0.60]\rangle,\langle 0.40,0.20\rangle)$ & $(\langle[0.25,0.30],[0.45,0.50]\rangle,\langle 0.60,0.30\rangle)$ & $(\langle[0.30,0.45],[0.20,0.25]\rangle,\langle 0.100 .80\rangle)$ & $(\langle[0.40,0.50],[0.10,0.30]\rangle,\langle 0.30,0.70\rangle)$ \\
\hline & $A_{4}$ & $(\langle[0.15,0.45],[0.25,0.30]\rangle,\langle 0.40,0.60\rangle)$ & $(\langle[0.20,0.25],[0.30,0.35]\rangle,\langle 0.15,0.20\rangle)$ & $(\langle[0.45,0.60],[0.20,0.25]\rangle,\langle 0.29,0.60\rangle)$ & $(\langle[0.16,0.20],[0.25,0.30]\rangle,\langle 0.15,0.30\rangle)$ \\
\hline & Weights & 0.20 & 0.25 & 0.15 & 0.40 \\
\hline \multirow{5}{*}{$D^{(3)}$} & $A_{1}$ & $(\langle[0.20,0.30],[0.25,0.40]\rangle,\langle 0.15,0.20\rangle)$ & $(\langle[0.30,0.35],[0.40,0.45]\rangle,\langle 0.40,0.30\rangle)$ & $(\langle[0.32,0.40],[0.35,0.45]\rangle,\langle 0.30,0.50\rangle)$ & $(\langle[0.15,0.18],[0.19,0.30]\rangle,\langle 0.30,0.60\rangle)$ \\
\hline & $A_{2}$ & $(\langle[0.30,0.50],[0.20,0.40]\rangle,\langle 0.10,0.30\rangle)$ & $(\langle[0.40,0.50],[0.10,0.30]\rangle,\langle 0.20,0.10\rangle)$ & $(\langle[0.40,0.45],[0.30,0.35]\rangle,\langle 0.60,0.20\rangle)$ & $(\langle[0.10,0.30],[0.20,0.50]\rangle,\langle 0.40,0.30\rangle)$ \\
\hline & $A_{3}$ & $(\langle[0.25,0.32],[0.40,0.45]\rangle,\langle 0.20,0.30\rangle)$ & $(\langle[0.30,0.35],[0.38,0.49]\rangle,\langle 0.20,0.62\rangle)$ & $(\langle[0.37,0.42],[0.20,0.29]\rangle,\langle 0.30,0.10\rangle)$ & $(\langle[0.20,0.35],[0.30,0.60]\rangle,\langle 0.20,0.42\rangle)$ \\
\hline & $A_{4}$ & $(\langle[0.40,0.44],[0.50,0.52]\rangle,\langle 0.30,0.20\rangle)$ & $(\langle[0.40,0.45],[0.35,0.40]\rangle,\langle 0.30,0.10\rangle)$ & $(\langle[0.10,0.18],[0.15,0.30]\rangle,\langle 0.40,0.50\rangle)$ & $(\langle[0.30,0.40],[0.50,0.55]\rangle,\langle 0.30,0.70\rangle)$ \\
\hline & Weights & 0.18 & 0.12 & 0.25 & 0.45 \\
\hline \multirow{5}{*}{$D^{(4)}$} & $A_{1}$ & $(\langle[0.30,0.40],[0.20,0.30]\rangle,\langle 0.40,0.60\rangle)$ & $(\langle[0.18,0.30],[0.19,0.34]\rangle,\langle 0.40,0.32\rangle)$ & $(\langle[0.30,0.38],[0.40,0.45]\rangle,\langle 0.30,0.40\rangle)$ & $(\langle[0.30,0.60],[0.20,0.40]\rangle,\langle 0.40,0.20\rangle)$ \\
\hline & $A_{2}$ & $(\langle[0.10,0.30],[0.20,0.50]\rangle,\langle 0.20,0.10\rangle)$ & $(\langle[0.25,0.29],[0.32,0.45]\rangle,\langle 0.60,0.10\rangle)$ & $(\langle[0.40,0.45],[0.47,0.50]\rangle,\langle 0.30,0.25\rangle)$ & $(\langle[0.10,0.15],[0.20,0.25]\rangle,\langle 0.30,0.50\rangle)$ \\
\hline & $A_{3}$ & $(\langle[0.20,0.31],[0.35,0.42]\rangle,\langle 0.30,0.10\rangle)$ & $(\langle[0.30,0.40],[0.52,0.59]\rangle,\langle 0.30,0.40\rangle)$ & $(\langle[0.18,0.36],[0.20,0.25]\rangle,\langle 0.20,0.40\rangle)$ & $(\langle[0.30,0.35],[0.40,0.45]\rangle,\langle 0.20,0.70\rangle)$ \\
\hline & $A_{4}$ & $(\langle[0.10,0.15],[0.30,0.40]\rangle,\langle 0.20,0.10\rangle)$ & $(\langle[0.20,0.30],[0.40,0.50]\rangle,\langle 0.20,0.50\rangle)$ & $(\langle[0.23,0.32],[0.40,0.45]\rangle,\langle 0.30,0.60\rangle)$ & $(\langle[0.16,0.32],[0.17,0.34]\rangle,\langle 0.30,0.40\rangle)$ \\
\hline & Weights & 0.35 & 0.40 & 0.12 & 0.13 \\
\hline
\end{tabular}
the previously obtained IVIFNs (from GD sessions)

Table 1. Rating values of each decision-maker in terms of CIFNs 
Table 2. Positive and negative ideals for each decision-maker.

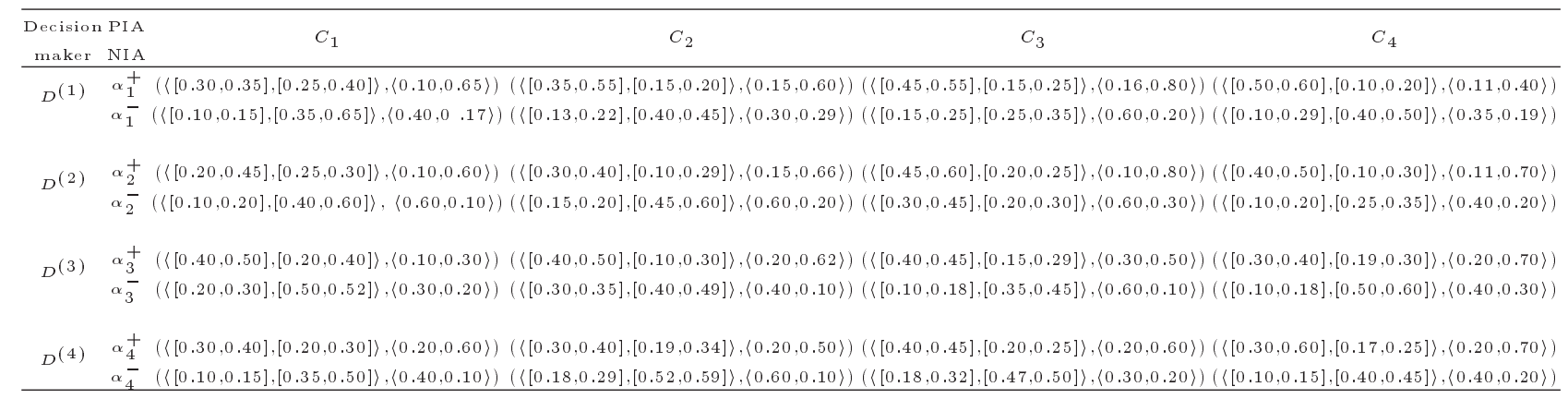

Table 3. Separation measures from ideal solutions corresponding to each decision-maker

\begin{tabular}{ccccccccc}
\hline \multirow{2}{*}{ Alternatives } & \multicolumn{2}{c}{$\boldsymbol{D}^{(\mathbf{1})}$} & \multicolumn{2}{c}{$\boldsymbol{D}^{(\mathbf{2})}$} & \multicolumn{2}{c}{$\boldsymbol{D}^{(\mathbf{3})}$} & \multicolumn{2}{c}{$\boldsymbol{D}^{(4)}$} \\
\cline { 2 - 8 } & $\boldsymbol{D}_{\boldsymbol{i}}^{(\mathbf{1}+)}$ & $\boldsymbol{D}_{\boldsymbol{i}}^{(\mathbf{1}-)}$ & $\boldsymbol{D}_{\boldsymbol{i}}^{(\mathbf{2}+)}$ & $\boldsymbol{D}_{\boldsymbol{i}}^{(\mathbf{2}-)}$ & $\boldsymbol{D}_{\boldsymbol{i}}^{(\mathbf{3}+)}$ & $\boldsymbol{D}_{\boldsymbol{i}}^{(\mathbf{3}-)}$ & $\boldsymbol{D}_{\boldsymbol{i}}^{(4+)}$ & $\boldsymbol{D}_{\boldsymbol{i}}^{(4-)}$ \\
\hline$A_{1}$ & 0.2167 & 0.1539 & 0.2344 & 0.1852 & 0.1361 & 0.1998 & 0.1359 & 0.2214 \\
& & & & & & & & \\
$A_{2}$ & 0.1921 & 0.1977 & 0.2166 & 0.1889 & 0.1899 & 0.1625 & 0.2378 & 0.1203 \\
& & & & & & & & \\
$A_{3}$ & 0.1082 & 0.2258 & 0.1966 & 0.2173 & 0.1658 & 0.1689 & 0.1909 & 0.1609 \\
$A_{4}$ & & & & & & & & \\
\hline
\end{tabular}

Table 4. Closeness coefficients and ranking order with respect to each decision-maker

\begin{tabular}{ccccccccc}
\hline & \multicolumn{2}{c}{$\boldsymbol{D}^{(\mathbf{1})}$} & \multicolumn{2}{c}{$\boldsymbol{D}^{(\mathbf{2})}$} & \multicolumn{2}{c}{$\boldsymbol{D}^{(\mathbf{3})}$} & \multicolumn{2}{c}{$\boldsymbol{D}^{(\mathbf{4})}$} \\
\hline Alternatives & $\mathfrak{C}_{\boldsymbol{i}}^{(\mathbf{1})}$ & $\mathbf{R a n k i n g}$ & $\mathfrak{C}_{\boldsymbol{i}}^{(\mathbf{2})}$ & Ranking & $\mathfrak{C}_{\boldsymbol{i}}^{(\mathbf{3})}$ & Ranking & $\mathfrak{C}_{\boldsymbol{i}}^{(4)}$ & Ranking \\
\hline$A_{1}$ & 0.4152 & 3 & 0.4414 & 4 & 0.5948 & 1 & 0.6196 & 1 \\
& & & & & & & & \\
$A_{2}$ & 0.5071 & 2 & 0.4659 & 3 & 0.4612 & 4 & 0.3359 & 4 \\
$A_{3}$ & 0.6761 & 1 & 0.5250 & 1 & 0.5047 & 3 & 0.4696 & 3 \\
$A_{4}$ & 0.3398 & 4 & 0.5016 & 2 & 0.5054 & 2 & 0.4912 & 2 \\
\hline
\end{tabular}

and IFNs (from the personal interview round) in the form of CIFNs;

Step 2: By using Eqs. (13) and (14), the ideal alternatives namely CIF-PIA and CIF-NIA, are determined for each decision-maker. The corresponding values are summarized in Table 2;

Step 3: Without loss of generality, we choose $q=2$ and compute the distance measure values by using Eq. (7) for each decision-maker; the obtained results are summarized in Table 3;

Step 4: Utilize Eq. (15) to compute the closeness coefficients with respect to each decision-maker. The results and the corresponding ranking order of the alternatives are summarized in Table 4, and observed that $A_{3}$ is the best candidate for the decision-makers $D^{(1)}$ and $D^{(2)}$ while $A_{1}$ for the other decision-makers;

Step 5: To overcome the ambiguity about the best alternatives with respect to the decision-makers, aggregate the ideal distance measurement values, as given in Table 3, of every decision-maker by using Eq. (16) corresponding to the five different priority pairs $\left(\lambda_{1}, \lambda_{2}, \lambda_{3}, \lambda_{4}\right)$ of decision-makers. The results are summarized in the fourth and fifth columns of Table 5;

Step 6: For each priority pair, the values of $\mathfrak{C}_{i}$ 's are computed by using Eq. (17), and their results are summarized in the sixth column of Table 5;

Step 7: Based on the values of $\mathfrak{C}_{i}$ 's, the ranking order of the alternatives is summarized in the last 
Table 5. Aggregated closeness coefficient and ranking for each candidate

\begin{tabular}{|c|c|c|c|c|c|c|c|c|}
\hline & \multirow{2}{*}{\multicolumn{2}{|c|}{$\begin{array}{r}\text { Weights } \\
\lambda \\
\end{array}$}} & \multirow[t]{2}{*}{ Candidates } & \multicolumn{2}{|c|}{ Distance measures } & \multirow[t]{2}{*}{$\mathfrak{C}_{\mathfrak{i}}$} & \multirow[t]{2}{*}{ Ranking } & \multirow{2}{*}{$\begin{array}{c}\text { Selected } \\
\text { candidate }\end{array}$} \\
\hline & & & & $D_{i}^{+}$ & $D_{i}^{-}$ & & & \\
\hline \multirow{4}{*}{ Case 1} & $D^{(1)}$ & 0.20 & $A_{1}$ & 0.1817 & 0.1884 & 0.5090 & 2 & \multirow{4}{*}{$A_{3}$} \\
\hline & $D^{(2)}$ & 0.30 & $A_{2}$ & 0.2031 & 0.1732 & 0.4603 & 4 & \\
\hline & $D^{(3)}$ & 0.40 & $A_{3}$ & 0.1660 & 0.1948 & 0.5399 & 1 & \\
\hline & $D^{(4)}$ & 0.10 & $A_{4}$ & 0.1980 & 0.1755 & 0.4699 & 3 & \\
\hline \multirow{4}{*}{ Case 2} & $D^{(1)}$ & 0.20 & $A_{1}$ & 0.1718 & 0.1963 & 0.5333 & 1 & \multirow{4}{*}{$A_{1}$} \\
\hline & $D^{(2)}$ & 0.20 & $A_{2}$ & 0.2148 & 0.1579 & 0.4237 & 4 & \\
\hline & $D^{(3)}$ & 0.20 & $A_{3}$ & 0.1705 & 0.1900 & 0.5271 & 2 & \\
\hline & $D^{(4)}$ & 0.40 & $A_{4}$ & 0.1988 & 0.1734 & 0.4660 & 3 & \\
\hline \multirow{4}{*}{ Case 3} & $D^{(1)}$ & 0.13 & $A_{1}$ & 0.1612 & 0.2007 & 0.5545 & 1 & \multirow{4}{*}{$A_{1}$} \\
\hline & $D^{(2)}$ & 0.15 & $A_{2}$ & 0.2143 & 0.1533 & 0.4170 & 4 & \\
\hline & $D^{(3)}$ & 0.30 & $A_{3}$ & 0.1735 & 0.1836 & 0.5142 & 2 & \\
\hline & $D^{(4)}$ & 0.42 & $A_{4}$ & 0.1933 & 0.1765 & 0.4773 & 3 & \\
\hline \multirow{4}{*}{ Case 4} & $D^{(1)}$ & 0.35 & $A_{1}$ & 0.1957 & 0.1827 & 0.4828 & 2 & \multirow{4}{*}{$A_{3}$} \\
\hline & $D^{(2)}$ & 0.32 & $A_{2}$ & 0.2074 & 0.1761 & 0.4592 & 3 & \\
\hline & $D^{(3)}$ & 0.16 & $A_{3}$ & 0.1598 & 0.2043 & 0.5612 & 1 & \\
\hline & $D^{(4)}$ & 0.17 & $A_{4}$ & 0.2091 & 0.1674 & 0.4446 & 4 & \\
\hline \multirow{4}{*}{ Case 5} & $D^{(1)}$ & 0.42 & $A_{1}$ & 0.2053 & 0.1774 & 0.4635 & 3 & \multirow{4}{*}{$A_{3}$} \\
\hline & $D^{(2)}$ & 0.36 & $A_{2}$ & 0.2052 & 0.1826 & 0.4708 & 2 & \\
\hline & $D^{(3)}$ & 0.12 & $A_{3}$ & 0.1552 & 0.2102 & 0.5753 & 1 & \\
\hline & $D^{(4)}$ & 0.10 & $A_{4}$ & 0.2139 & 0.1643 & 0.4343 & 4 & \\
\hline
\end{tabular}

Table 6. Rating values of the worse alternative, $A_{1}^{\prime}$, for each decision-maker

\begin{tabular}{ccc}
\hline $\begin{array}{c}\text { Decision } \\
\text { maker }\end{array}$ & $C_{1}$ & $C_{2}$ \\
\hline$D^{(1)}$ & $(\langle[0.15,0.20],[0.30,0.45]\rangle,\langle 0.25,0.50\rangle)(\langle[0.13,0.20],[0.40,0.48]\rangle,\langle 0.35,0.50\rangle)(\langle[0.30,0.35],[0.25,0.35]\rangle,\langle 0.60,0.30\rangle)(\langle[0.10,0.20],[0.20,0.35]\rangle,\langle 0.15,0.10\rangle)$ \\
$D^{(2)}$ & $(\langle[0.10,0.15],[0.30,0.45]\rangle,\langle 0.62,0.05\rangle)(\langle[0.15,0.18],[0.25,0.35]\rangle,\langle 0.20,0.50\rangle)(\langle[0.44,0.48],[0.20,0.35]\rangle,\langle 0.20,0.30\rangle)(\langle[0.10,0.25],[0.20,0.39]\rangle,\langle 0.20,0.10\rangle)$ \\
$D^{(3)}$ & $(\langle[0.20,0.25],[0.25,0.42]\rangle,\langle 0.20,0.15\rangle)(\langle[0.32,0.35],[0.40,0.50]\rangle,\langle 0.44,0.20\rangle)(\langle[0.32,0.38],[0.35,0.50]\rangle,\langle 0.40,0.20\rangle)(\langle[0.15,0.17],[0.19,0.32]\rangle,\langle 0.35,0.50\rangle)$ \\
$D^{(4)}$ & $(\langle[0.30,0.35],[0.20,0.35]\rangle,\langle 0.50,0.40\rangle)(\langle[0.18,0.25],[0.19,0.39]\rangle,\langle 0.50,0.30\rangle)(\langle[0.30,0.35],[0.40,0.50]\rangle,\langle 0.40,0.20\rangle)(\langle[0.30,0.50],[0.20,0.45]\rangle,\langle 0.45,0.15\rangle)$ \\
\hline
\end{tabular}

column of Table 5. From this table, we can see that concerning the different pairs, the best alternative is either $A_{1}$ or $A_{3}$.

\subsection{Validity test}

The following test criteria are presented by Wang and Triantaphyllou [9] to validate the approach:

Test Criterion 1: If we replace the rating values of non-optimal alternative with a worse alternative, then the best alternative should not change, provided the relative weighted criteria remain unchanged;

Test Criterion 2: Method should possess a transitive nature;

Test Criterion 3: When a given problem is de- composed into smaller ones and the same MCDM method is applied, then the combined ranking of the alternatives should be identical to the ranking of the un-decomposed one.

Below, we have validated these test criteria by our proposed method.

\subsubsection{Validity test by Test Criterion 1}

Without loss of generality, we have considered Case 5 of the above-discussed analysis (similarly for the other cases as given in Table 5), where the priority level of the decision-makers has been taken as $0.42,0.36$, $0.12,0.10$, respectively. The original ranking order for the case is $A_{3} \succ A_{2} \succ A_{1} \succ A_{4}$. Now, in order to validate it with respect to Criterion 1 , the following decision-makers, given in Table 6 , are obtained from the original matrices after replacing the non-optimal 
Table 7. Comparison analysis with some of the existing approaches

\begin{tabular}{lccccc}
\hline Existing & \multicolumn{9}{c}{ Aggregated closeness coefficients } & Ranking \\
\cline { 2 - 5 } approaches & $\mathfrak{C}_{1}$ & $\mathfrak{C}_{2}$ & $\mathfrak{C}_{3}$ & $\mathfrak{C}_{4}$ & \\
\hline Fahmi et al. [40] & 0.4350 & 0.5103 & 0.5618 & 0.4693 & $A_{3} \succ A_{2} \succ A_{4} \succ A_{1}$ \\
Lu and Ye [30] & 0.5293 & 0.4911 & 0.4829 & 0.5171 & $A_{3} \succ A_{2} \succ A_{4} \succ A_{1}$ \\
Biswas and Kumar [25] & 0.5471 & 0.5729 & 0.5867 & 0.5553 & $A_{3} \succ A_{2} \succ A_{4} \succ A_{1}$ \\
& & & & & \\
Gupta et al. [34] & 0.5648 & 0.5021 & 0.4453 & 0.5356 & $A_{3} \succ A_{2} \succ A_{4} \succ A_{1}$ \\
& & & & & \\
Dugenci [21] & 0.3510 & 0.5396 & 0.5803 & 0.4187 & $A_{3} \succ A_{2} \succ A_{4} \succ A_{1}$ \\
& & & & & \\
Wang and Chen [33] & 0.5300 & 0.4917 & 0.4865 & 0.5161 & $A_{3} \succ A_{2} \succ A_{4} \succ A_{1}$ \\
\hline
\end{tabular}

alternative $\left(A_{1}\right)$ with an arbitrary worst alternative $\left(A_{1}^{\prime}\right)$.

Then, by applying the proposed approach to this data closeness coefficients, $\mathfrak{C}_{i}$ 's, of each candidate, $A_{i}(i=1,2,3,4)$, are obtained as $0.3601,0.5246,0.5465$, and 0.4111 . Thus, the ranking order of the candidate is $A_{3} \succ A_{2} \succ A_{4} \succ A_{1}^{\prime}$, which shows that the best alternative remains the same, i.e. $A_{3}$.

\subsubsection{Validity test by Test Criteria 2 and 3}

Under this test, if the given problem is decomposed into sub-problems, namely $\left\{A_{2}, A_{3}, A_{1}\right\},\left\{A_{2}, A_{3}, A_{4}\right\}$, and $\left\{A_{3}, A_{1}, A_{4}\right\}$, and the same procedure steps of the approach are applied, then we get the ranking orders of these sub-problems as $A_{3} \succ A_{1} \succ A_{2}$, $A_{3} \succ A_{2} \succ A_{4}$, and $A_{3} \succ A_{1} \succ A_{4}$, respectively. Therefore, by combining these, we obtain the overall ranking order of the alternative as $A_{3} \succ A_{1} \succ A_{2} \succ A_{4}$, which is the same as that of the original ranking order; hence, it characterizes the transitive property. Thus, the proposed approach is valid under test Criteria 2 and 3 .

\subsection{Comparative studies}

In order to compare the serformances of the proposed approach with respect to the existing approaches $[21,25,30,33,34,40]$ under the CFSs, IVIFSs, IFSs, and interval-valued FSs environments, an analysis has been conducted. To apply these existing approaches to the considered data, first, the rating values of CIFNs are converted into these numbers by taking the rating corresponding to IFNs as zero. Further, without loss of generality, a case is considered by taking the weight vector of the decision-makers as $\lambda=(0.42,0.36,0.12,0.10)^{T}$ and, hence, the existing approaches are applied to the considered data. The results computed by these different approaches are summarized in Table 7 , and it is concluded that the ranking order of the given alternatives is $A_{3} \succ A_{2} \succ$ $A_{4} \succ A_{1}$; hence, the best alternative is $A_{3}$ that is in agreement with the proposed approach results given in Table 5, validating the stability of our approach.

According to the result of comparing these existing approaches with general intuitionistic sets (IVIFSs or IFSs), the proposed DM method under the CIFS environment contains much more evaluation information on the alternatives by considering both the IVIFSs and IFSs simultaneously, while the existing approaches contain either IFS or IVIFS information. Therefore, the approaches under the IVIFSs or IFSs may lose some useful information, either IVIFNs or IFNs, of alternatives which may affect the decision results. Furthermore, it is noted from the study that the computational procedure of the proposed approach is different from the existing approaches under the different environment; however, the proposed result in this paper is more rational to reality in the decision process due to the consideration of the consistent priority degree between the pairs of the arguments and between different experts. Moreover, the corresponding studies under the IVIFS or IFS environment can be considered as the special case of the proposed operators. Finally, the existing DM methods under IVIFSs or IFSs cannot deal with the DM problem by CIFS. Therefore, the proposed approach is more generalized and suitable to capture the real-life fuzziness more accurately than the existing ones.

In addition some characteristics of the proposed method are compared with those of the aforementioned methods, as listed in Table 8.

\section{Conclusion}

CIFS is one of the successful extensions of the IFS in 
Table 8. The comparative characteristics of different methods.

\begin{tabular}{lcccc}
\hline & $\begin{array}{c}\text { Whether flexible to } \\
\text { express a wider range } \\
\text { of information }\end{array}$ & $\begin{array}{c}\text { Whether considering Whether describing Whether having the } \\
\text { more than one } \\
\text { Methods }\end{array}$ & $\checkmark$ & $\begin{array}{c}\text { hybrid information } \\
\text { characteristic of }\end{array}$ \\
\hline Lu and Ye [30] & $\checkmark$ & $\times$ & $\times$ & $\times$ \\
at the same level & $\times$ \\
Wang and Chen [33] & $\checkmark$ & $\checkmark$ & $\times$ & $\times$ \\
Gupta et al. [34] & $\checkmark$ & $\checkmark$ & $\times$ & $\times$ \\
Dugenci [21] & $\checkmark$ & $\times$ & $\times$ & $\times$ \\
Biswas and Kumar [25] & $\checkmark$ & $\checkmark$ & $\checkmark$ & $\times$ \\
Fahmi et al. [40] & $\checkmark$ & $\checkmark$ & & $\times$ \\
The proposed method & & & $\times$
\end{tabular}

which a degree of the disagreement (in the form of IFS values) corresponding to the agreed interval region (in form of IVIFS) was used to represent the data. Considering its advantages, this study presents an extended TOPSIS approach to solve the group Decision Making (DM) problems under the CIFS environment. To this end, some generalized distance measures between the pairs of the CIF numbers were proposed. The prominent characteristic of these distance measures was also studied. Then, based on these measures, an extended TOPSIS group DM approach was presented for solving MCGDM problem under CIFS environment. The proposed approach was illustrated with a numerical example, and their results were compared with some of the existing approaches. In addition, the characteristics of the proposed approach comparable to those of the existing approaches were summarized. From this study, it was obtained that the several approaches under CFSs, IVIFSs, and/or IFSs were the special cases of the proposed approach. Thus, the proposed approach is more generalized and suitable to capture the real-life fuzziness more accurately than the existing ones. In the future, the result of this paper can be extended to the Pythagorean fuzzy environment and other uncertain and fuzzy environments [12,35,43-45].

\section{References}

1. Garg, H. and Arora, R. "Dual hesitant fuzzy soft aggregation operators and their application in decision making", Cognitive Computation, 10(5), pp. 769-789 (2018).

2. Garg, H. "Some arithmetic operations on the generalized sigmoidal fuzzy numbers and its application", Granular Computing, 3(1), pp. 9-25 (2018).

3. Zadeh, L.A. "Fuzzy sets", Information and Control, 8, pp. 338-353 (1965).

4. Atanassov, K.T. "Intuitionistic fuzzy sets", Fuzzy Sets and Systems, 20, pp. 87-96 (1986).
5. Atanassov, K. and Gargov, G. "Interval-valued intuitionistic fuzzy sets", Fuzzy Sets and Systems, 31, pp. 343-349 (1989).

6. Arora, R. and Garg, H. "Robust aggregation operators for multi-criteria decision making with intuitionistic fuzzy soft set environment", Scientia Iranica, E, 25(2), pp. 931-942 (2018).

7. Bagheri, M., Shojaei, P., and Khorami, M. "A comparative survey of the condition of tourism infrastructure in Iranian provinces using VIKOR and TOPSIS", Decision Science Letters, 7(1), pp. 87-102 (2018).

8. Garg, H. and Ansha, "Arithmetic operations on generalized parabolic fuzzy numbers and its application", Proceedings of the National Academy of Sciences, India Section A: Physical Sciences, 88(1), pp. 15-26 (2018).

9. Wang, X. and Triantaphyllou, E. "Ranking irregularities when evaluating alternatives by using some ELECTRE methods", Omega - International Journal of Management Science, 36, pp. 45-63 (2008).

10. Peng, X.D. and Garg, H. "Algorithms for intervalvalued fuzzy soft sets in emergency decision making based on WDBA and CODAS with new information measure", Computers \& Industrial Engineering, 119, pp. 439-452 (2018).

11. Xu, Z.S. and Yager, R.R. "Some geometric aggregation operators based on intuitionistic fuzzy sets", International Journal of General Systems, 35, pp. 417-433 (2006).

12. Peng, X.D. and Selvachandran, G. "Pythagorean fuzzy set: state of the art and future directions", Artificial Intelligence Review, 52, pp. 1873-1927 (2019).

13. Arora, R. and Garg, H. "Prioritized averaging/geometric aggregation operators under the intuitionistic fuzzy soft set environment", Scientia Iranica, E, 25(1), pp. 466-482 (2018).

14. Peng, X.D. "New operations for interval-valued Pythagorean fuzzy set", Scientia Iranica, E, 26(2), pp. 1049-1076 (2019). 
15. Garg, H. and Kumar, K. "Some aggregation operators for linguistic intuitionistic fuzzy set and its application to group decision-making process using the set pair analysis", Arabian Journal for Science and Engineering, 43(6), pp. 3213-3227 (2018).

16. Garg, H. "Linguistic Pythagorean fuzzy sets and its applications in multiattribute decision-making process", International Journal of Intelligent Systems, 33(6), pp. 1234-1263 (2018).

17. Hwang, C.L. and Yoon, K., Multiple Attribute Decision Making Methods and Applications A State-of-the-Art Survey, Springer-Verlag Berlin Heidelberg (1981).

18. Szmidt, E. and Kacprzyk, J. "Distances between intuitionistic fuzzy sets", Fuzzy Sets and Systems, 114, pp. 505-518 (2000).

19. Hung, W.L. and Yang, M.S. "Similarity measures of intuitionistic fuzzy sets based on hausdorff distance", Pattern Recognition Letters, 25, pp. 1603-1611 (2004).

20. Boran, F.E., Genç S., and Akay, D. "Personnel selection based on intuitionistic fuzzy sets", Human Factors and Ergonomics in Manufacturing \& Service Industries, 21(5), pp. 493-503 (2011).

21. Dugenci, M. "A new distance measure for interval valued intuitionistic fuzzy sets and its application to group decision making problems within complete weights information", Applied Soft Computing, 41, pp. 120-134 (2016).

22. Garg, H. "A new generalized improved score function of interval-valued intuitionistic fuzzy sets and applications in expert systems", Applied Soft Computing, 38, pp. 988-999 (2016).

23. Mohammadi, A., Shojaei, P., Kaydan, B., and Akbari, Z. "Prioritizing the performance of civil development projects in governmental administration agencies, using gray relational analysis (GRA) and TOPSIS approach", Decision Science Letters, 5(4), pp. 487-498 (2016).

24. Garg, H., Agarwal, N., and Tripathi, A. "Generalized intuitionistic fuzzy entropy measure of order $\alpha$ and degree $\beta$ and its applications to multi-criteria decision making problem", International Journal of Fuzzy System Applications, 6(1), pp. 86-107 (2017).

25. Biswas, A. and Kumar, S. "An integrated TOPSIS approach to MADM with interval-valued intuitionistic fuzzy settings", in: Advanced Computational and Communication Paradigms, Springer, pp. 533-543 (2018).

26. Vommi, V. "TOPSIS with statistical distances: A new approach to MADM", Decision Science Letters, 6(1), pp. 49-66 (2017).

27. Singh, S. and Garg, H. "Distance measures between type-2 intuitionistic fuzzy sets and their application to multicriteria decision-making process", Applied Intelligence, 46(4), pp. 788-799 (2017).

28. Li, D.F. "TOPSIS- based nonlinear-programming methodology for multiattribute decision making with interval-valued intuitionistic fuzzy sets", IEEE Transactions on Fuzzy Systems, 18, pp. 299-311 (2010).
29. Garg, H. and Arora, R. "A nonlinear-programming methodology for multi-attribute decision-making problem with interval-valued intuitionistic fuzzy soft sets information", Applied Intelligence, 48(8), pp. 20312046 (2018).

30. Lu, Z. and Ye, J. "Logarithmic similarity measure between interval-valued fuzzy sets and its fault diagnosis method", Information, 9(2), p. 36 (2018). DOI: 10.3390/info9020036

31. Garg, H. and Kumar, K. "An advanced study on the similarity measures of intuitionistic fuzzy sets based on the set pair analysis theory and their application in decision making", Soft Computing, 22(15), pp. 49594970 (2018).

32. Askarifar, K., Motaffef, Z., and Aazaami, S. "An investment development framework in Iran's seashores using TOPSIS and best-worst multi-criteria decision making methods", Decision Science Letters, 7(1), pp. 55-64 (2018).

33. Wang, C.Y. and Chen, S.-M. "A new multiple attribute decision making method based on intervalvalued intuitionistic fuzzy sets, linear programming methodology, and the TOPSIS method", in: Advanced Computational Intelligence (ICACI), 2017 Ninth International Conference on, IEEE, pp. 260-263 (2017).

34. Gupta, P., Mehlawat, M.K., Grover, N., and Pedrycz, W. "Multi-attribute group decision making based on extended TOPSIS method under interval-valued intuitionistic fuzzy environment", A pplied Soft Computing, 69, pp. 554-567 (2018).

35. Kumar, K. and Garg, H. "TOPSIS method based on the connection number of set pair analysis under interval-valued intuitionistic fuzzy set environment", Computational and Applied Mathematics, 37(2), pp. 1319-1329 (2018).

36. Kumar, K. and Garg, H. "Connection number of set pair analysis based TOPSIS method on intuitionistic fuzzy sets and their application to decision making", Applied Intelligence, 48(8), pp. 2112-2119 (2018).

37. Jun, Y.B., Kim, C.S., and Yang, K.O. "Cubic sets", Annals of Fuzzy Mathematics and Informatics, 4(1), pp. 83-98 (2012).

38. Khan, M., Abdullah, S., Zeb, A., and Majid, A. "Cubic aggregation operators", International Journal of Computer Science and Information Security, 14(8), pp. $670-682(2016)$.

39. Mahmood, T., Mehmood, F., and Khan, Q. "Some generalized aggregation operators for cubic hesitant fuzzy sets and their applications to multi criteria decision making", Journal of Mathematics, 49(1), pp. 31-49 (2017).

40. Fahmi, A., Abdullah, S., Amin, F., and Ali, A. "Precursor selection for sol-gel synthesis of titanium carbide nanopowders by a new cubic fuzzy multiattribute group decision-making model", Journal of Intelligent Systems, 28(5), pp. 699-720 (2019). 
41. Kaur, G. and Garg, H. "Multi-attribute decisionmaking based on bonferroni mean operators under cubic intuitionistic fuzzy set environment", Entropy, 20(1), p. 65 (2018). DOI: 10.3390/e20010065

42. Kaur, G. and Garg, H. "Cubic intuitionistic fuzzy aggregation operators", International Journal for Uncertainty Quantification, 8(5), pp. 405-428 (2018).

43. Garg, H. "New exponential operational laws and their aggregation operators for interval-valued pythagorean fuzzy multicriteria decision-making", International Journal of Intelligent Systems, 33(3), pp. 653-683 (2018).

44. Garg, H. "Hesitant Pythagorean fuzzy sets and their aggregation operators in multiple attribute decision making", International Journal for Uncertainty Quantification, 8(3), pp. 267-289 (2018).

45. Garg, H. and Rani, D. "Some generalized complex intuitionistic fuzzy aggregation operators and their application to multicriteria decision-making process", Arabian Journal for Science and Engineering, 44(3), pp. 2679-2698 (2019).

\section{Biographies}

Harish Garg associated with the School of Mathematics at Thapar Institute of Engineering \& Technology (Deemed University) Patiala, India as an Assistant Professor. Prior to joining this University, he received an MSc in Mathematics from Punjabi University, Patiala, India, in 2008 and a PhD in Applied Mathematics with specialty in Reliability Theory and Soft Computing Techniques from Indian Institute of Technology,
Roorkee, India, in 2013. His research interests are in the fields of computational intelligence, multicriteria decision making problems, and fuzzy and intuitionistic fuzzy sets theories, Pythagorean fuzzy sets, Computing with words and Soft Computing. Dr. Garg has authored/co-authored over 215 technical papers published in refereed International Journals. Also, he has published 7 book chapters. His biographical profile has been selected for inclusion in the International Who's Who of Professionals, Marquis Who's Who in the World, and Marquis Who's Who in Science and Engineering. He is the Associate Editor for Journal of Intelligent \& Fuzzy Systems, International Journal of Computational Intelligence Systems, Technological and Economic Development of Economy, Mathematical Problems in Engineering, Journal of Industrial \& Management Optimization, Complexity, Complex and Intelligent Systems, CAAI Transactions on Intelligence Technology and so on. His Google citations are over $6440+$.

Gagandeep Kaur received the MSc degree in Applied Mathematics and Computing from Department of Mathematics, Punjabi University Patiala, Punjab, India, in 2016. Presently, she is working towards her $\mathrm{PhD}$ degree in Thapar Institute of Engineering \& Technology (Deemed University) Patiala, India under the guidance of Dr. Harish Garg. Her current research interests are in the area of multi criteria decision-making, aggregation operator, intuitionistic fuzzy set. She has published 10 papers in the reputed international journals. 